\title{
Thinning and expansion of the substorm plasma sheet: Cluster PEACE timing analysis
}

\author{
J. P. Dewhurst ${ }^{1}$, C. J. Owen ${ }^{1}$, A. N. Fazakerley ${ }^{1}$, and A. Balogh ${ }^{2}$ \\ ${ }^{1}$ Mullard Space Science Lab., University College London, Holmbury-St. Mary, Dorking, Surrey, RH5 6NT, UK \\ ${ }^{2}$ Imperial College London, South Kensington, London, SW7 2AZ, UK
}

Received: 19 January 2004 - Revised: 6 August 2004 - Accepted: 15 August 2004 - Published: 22 December 2004

\begin{abstract}
The storage and subsequent removal of magnetic flux in the magnetotail during a geomagnetic substorm has a dramatic effect on the thickness of the cross-tail plasma sheet. The near-Earth plasma sheet is thought to thin during the growth phase and then rapidly expand after onset of the substorm. The direction of propagation, whether earthward or tailward along the GSM-X direction in the near-Earth tail, may suggest the time ordering of current-disruption and near-Earth reconnection, both of which are key to the substorm process. Cluster's Plasma Electron And Current Experiment (PEACE) allows 4-point observations of electrons at the plasma sheet - lobe boundary as this interface passes over the Cluster tetrahedron. The relative timings of the boundary passage at each spacecraft allow a determination of this boundary's speed and direction of motion, assuming this is planar on the scale of the Cluster separation scale. For those boundaries corresponding to the expansion of the plasma sheet, this direction is fundamental to determining the direction of expansion. We present an example of isolated thinning and expansion of the plasma sheet, as well as a multiple thinning-expansion event that occurs during a more active substorm. Data from the 2001 and 2002 tail passes have been analysed and the average plasma sheet - lobe boundary normal vectors and normal component velocities have been calculated. A total of 77 crossings, typically between 10 and $20 \mathrm{R}_{E}$ downtail, correspond to substorm associated expansion of the plasma sheet over the spacecraft. These had normal vectors predominantly in the GSM-YZ plane and provided no clear evidence for the formation of the near-Earth neutral line occurring before current disruption or vice versa. The expansions of the plasma sheet generally exhibit the appropriate GSM-Z direction expected for the given lobe, and tend to have GSM-Y components that support onset occurring near the origin of the GSM-YZ plane. This result is noteworthy in that it indicates a homogeneous plasma sheet expansion. These expansions have an average velocity along their normal of $60 \pm 37 \mathrm{~km} \mathrm{~s}^{-1}$. Conversely we find an average thinning velocity of $43 \pm 32 \mathrm{~km} \mathrm{~s}^{-1}$ from 66 substorm-
\end{abstract}

Correspondence to: J. P. Dewhurst

(jpd@mssl.ucl.ac.uk) associated thinnings. The normal vectors of the thinning plasma sheet vary considerably in the GSM-YZ plane across the entire magnetotail, suggesting that more complex dynamics govern this process.

Key words. Magnetospheric physics (Magnetotail; Plasma sheet; Storms and substorms)

\section{Introduction}

Magnetospheric substorms (Akasofu, 1968) are disturbances in the magnetosphere and ionosphere that result from enhanced coupling between the solar wind and the magnetosphere. These are observed as fluctuations of the terrestrial magnetic field measured at high-latitudes on the ground (Akasofu and Chapman, 1961) and are associated with auroral signatures (Akasofu, 1964). It was found that during substorm activity the magnetotail plasma sheet exhibits contractions and expansions associated with the addition and removal of magnetic flux to and from the magnetotail (Bame et al., 1967).

Auroral substorms were shown to develop in the following order: the growth phase; the onset of the substorm; expansion poleward and in local time; and finally, recovery to the pre-substorm state (Akasofu, 1964). Magnetospheric and ionospheric changes occur, corresponding to each auroral substorm phase, particularly in the magnetotail (e.g. McPherron, 1970; Aubry and McPherron, 1971; Russell and McPherron, 1973).

The growth phase of a substorm begins when the interplanetary magnetic field (IMF) turns southward, forming the dayside magnetopause neutral line. Enhanced magnetic reconnection occurs, leading to the rapid erosion of dayside magnetic flux (Maezawa, 1975; Baker et al., 1984). Reconnected magnetic flux is transported over the poles and stored in the magnetotail. The flux added to the magnetotail during the growth phase causes an increase in the tail radius and an increase in the magnetopause flaring angle (Coroniti and Kennel, 1972; Fairfield, 1985). This, in turn, allows the solar wind to exert a greater pressure on the magnetopause. 
To maintain overall pressure balance, the magnetic pressure of the lobes increases (e.g. Fairfield and Ness, 1970) and the plasma sheet tends to thin with an increase in plasma pressure (e.g. Axford et al., 1965). This process is dynamic and continually adjusts to maintain a pressure balance. This compression of the plasma sheet during the growth phase of a substorm was first observed by the VELA 2A and 2B satellites (Hones et al., 1967). Subsequently, observational evidence of central plasma sheet thinning associated with a southward turning of the IMF was reported by Hones et al. (1971a) and Aubry and McPherron (1971). Thinning in the dawn and dusk flanks of the tail is generally not as pronounced as in the midnight sector, suggesting that plasma sheet plasma and magnetic flux may be forced towards the flanks in the process (Hones et al., 1971a).

The growth phase ends with the onset of a substorm, representing a rapid release of the stored energy and characterized by the sudden brightening of the most equatorward auroral arc (Akasofu, 1964; Rostoker et al., 1980). The auroral brightening is associated with the diversion of the enhanced cross-tail current (McPherron et al., 1973) along magnetic field lines and into the ionosphere, forming the substorm current wedge (Bonnevier and Rostoker, 1970; McPherron et al., 1973; Rostoker, 1974). The associated dipolarization of the magnetic field that occurs at substorm onset has been well documented (e.g. Cummings et al., 1968; Kokubun and McPherron, 1981). In the near-Earth magnetotail the formation of a neutral line during substorms is inferred from observations of fast flows forming bursty-bulk flows (BBFs) (e.g. Angelopoulos et al., 1996). This Near-Earth Neutral Line (NENL) is formed in the late growth and early expansion phases in the thinned plasma sheet (Hones, 1973; McPherron et al., 1973; Russell and McPherron, 1973). Closed plasma sheet field lines are reconnected first, followed by the open field lines in the lobes. Earthward of the new neutral line the newly closed field lines contract earthward due to their magnetic tension. These returning field lines become more dipolar as they propagate towards the Earth. Tailward of the NENL, reconnected closed plasma sheet field lines are pinched off and form a plasmoid: a closed loop or tightly wound helical structure around an O-line in which the magnetic field is neither connected to the Earth nor the solar wind (Hones et al., 1984a).

The two most developed models of substorm activity at and after auroral onset suggest onset is initiated by either the formation of a NENL (see review by Baker et al., 1996) or by current disruption closer to Earth (see review by Lui et al., 1996). However, other models exist and are summarized by Rostoker et al. (1996). The initiating process is then the cause of the other observed phenomena. Various timing analyses have been performed to try and identify the initiator of the magnetospheric substorm (e.g. Kepko et al., 2004; Baker et al., 2002; Ohtani et al., 1999). Whilst Ohtani et al. (1999) suggest that current disruption need not follow directly from NENL formation, Kepko et al. (2004) and Baker et al. (2002) conclude that a flow-burst driven model, i.e. flow from a NENL, best describes their selected case studies. Liou et al. (1999) show that there is a need for intercalibration of the various data sets used for such timing work, to help decipher the data.

One aspect of the magnetospheric dynamics that may help resolve the issue of substorm onset initiator is the direction of the plasma sheet expansion. Many single spacecraft missions and fortuitous spacecraft conjunctions made observations of plasma sheet thinning and expansion (e.g. Hones et al., 1984b; Dandouras et al., 1986; Pytte et al., 1978; Baumjohann et al., 1992). These results provide information on the possible location of the NENL, but the lack of spatial and temporal resolution limits further knowledge of the large-scale dynamics.

Data from ESA's Cluster mission allows resolution between temporal and spatial phenomena of the tail plasma sheet and its environment in the region $\sim-15>\mathrm{X}_{\mathrm{GSM}}>-20 \mathrm{R}_{E}$. By performing timing analysis on data from Cluster's Plasma Electron And Current Experiment (PEACE), taken from the first two tail seasons, July through to November of 2001 and 2002, we determine the directions and normal component velocities of the plasma sheet - lobe boundary as it expands or contracts over the spacecraft quartet during the course of substorms. This allows us to determine any bias of the plasma sheet thinning and/or expansion direction vector and to consider whether these provide supporting evidence for either the NENL or current disruption models of substorms.

The Cluster PEACE instruments and the data products used here are described in the next section. In Sect. 3 the timing analysis method used is explained. A case study of a plasma sheet thinning and expansion is discussed in Sect. 4.1 and is accompanied by an example of multiple thinningexpansion events occurring during a complex substorm in Sect. 4.2. Survey statistics are provided in Sect. 4.3. Observations of pairs of thinnings and expansions are detailed in Sect. 4.4. Finally, these results are discussed and the complex nature of the plasma sheet is illustrated.

\section{Instrumentation and data}

ESA's Cluster mission was conceived as a means to resolve temporal from spatial phenomena in and around the magnetosphere. For example, the four-point measurements allow the determination of the orientation of the interface between adjacent plasma populations, together with the velocity and acceleration of this boundary as it passes over the quartet. The four spacecraft are equipped with identical payloads to provide the four-point measurements of particle populations, magnetic and electric fields and the oscillations of these fields.

The four Cluster spacecraft, in a polar orbit with an apogee of approximately $20 \mathrm{R}_{E}$, perform north-to-south passages through the nightside magnetosphere. Propagation of the spacecraft apogee through the magnetotail, due to the natural precession of the orbit, occurs between mid-July and midNovember. The spacecraft thus traversed the plasma sheet 
on approximately 100 orbits in the 2001 and 2002 tail season data sets used in this study.

The Plasma Electron And Current Experiment (PEACE) on board each spacecraft detects electrons in the energy range $0.59 \mathrm{eV}-26.5 \mathrm{keV}$ (Johnstone et al., 1997). Each PEACE instrument consists of two top-hat electrostatic plate analysers: the High Energy Electron Analyser (HEEA) and the Low Energy Electron Analyser (LEEA). Both detectors are capable of detecting electrons over the whole energy range, although they typically operate in two overlapping energy range subsets of the energy range given above. The HEEA and LEEA detectors differ only by their sensitivity to particular energies of electrons.

During normal operation, the spacecraft provides insufficient telemetry to allow for the transmission of the full threedimensional electron distributions for each four-second spin of the spacecraft. Thus, the instrument routinely returns pitch angle distributions and velocity space moments that are produced by onboard algorithms. Periods of burst mode telemetry are possible, during which 3-D distributions may also be telemetered to the ground, and are generally scheduled for short intervals (a few hours) around the expected encounter with a target boundary crossing such as the tail neutral sheet (Hapgood et al., 1997).

The Active Spacecraft POtential Control (ASPOC) instrument is designed to maintain the potential of each spacecraft below $10 \mathrm{~V}$ (Reidler et al., 1997) and a spacecraft potential of $+10 \mathrm{~V}$ was assumed for the onboard electron moment calculation routine performed by the PEACE instrument. However, ASPOC failed on one of the spacecraft during the instrument-commissioning phase and the other ASPOC units are operated only on selected orbits. The consequence of this situation is that the spacecraft potential may, at times, rise well above the $10 \mathrm{~V}$ threshold such that the PEACE onboard moment's calculation is seriously contaminated by the inclusion of spacecraft photoelectrons in the calculation. These photoelectrons may even enter the higher energy range of the HEEA sensor (generally $>34 \mathrm{eV}$ ) when the spacecraft are located in the tail lobes. In this region, low plasma densities generally result in the highest levels of spacecraft charging which prevent photoelectrons from escaping and significantly accelerate any natural electrons as they enter the detectors. Details of the effects of ASPOC on PEACE data are illustrated by Szita et al. (2001).

Magnetic field data from the Flux-Gate Magnetometer (FGM) instrument (Balogh, 1997) are used to determine spacecraft location north or south of the cross-tail current sheet and as an aid to substorm activity identification.

\section{Methodology}

There are several methods for the analysis of boundaries between different fields and plasma populations using multispacecraft data (Dunlop and Woodward, 1999; Harvey, 2000; Haaland et al., 2004). Dunlop and Woodward (1999) make use of four-point magnetic field minimum variance analy- sis vectors to obtain a description of non-planar, accelerating boundaries. The local normal vector of the boundary can be calculated at each point, and the variation of this normal through space and with time can be assessed. However, the signatures of the spacecraft transition between the lobe and the plasma sheet, which are the prime interest in this study, are generally observed as a change of plasma properties rather than magnetic field direction. Therefore, the Dunlop and Woodward (1999) method is not adopted in this study.

A boundary analysis method that considers the properties of the transition boundary layer between two plasma regions in detail is described by Haaland et al. (2004). This method assumes that the interface maintains a constant thickness during passage across the four spacecraft, and thus variations in its profile can be de-convolved into accelerations of the boundary.

As there is a major contrast in density between the plasma sheet and the lobes, and given that the PEACE data set used in this study has a temporal resolution of $4 \mathrm{~s}$ (suggesting a timing an error of $\pm 2 \mathrm{~s}$ which is short compared to a typical time lag of $\sim 10$ time stamps or $40 \mathrm{~s}$ ), we believe that a simpler method can be used with a basic correlation technique to provide the required boundary parameters to a good accuracy. Assuming that the boundary between the plasma sheet and the lobe can be represented as a plane moving at constant velocity, its characteristic unit normal $\hat{n}$ and velocity $V$ along that normal can be calculated (Harvey, 2000). An unambiguous boundary is represented by a repeatable and observable change in a given plasma parameter observed to pass over each of the four spacecraft at a time $t_{\alpha}$ with no possibility of inconsistency, as illustrated in Fig. 1. In this figure, the Cluster spacecraft are represented by $\mathrm{C} 1, \mathrm{C} 2, \mathrm{C} 3$ and $\mathrm{C} 4$ and are shown in a typical orientation for the midseason tail apogee pass with respect to the indicated GSM co-ordinate system. For each spacecraft, a generic plasma parameter trace is shown at the bottom of the figure and corresponds to the changes observed as a boundary between two plasma population passes over the spacecraft in the direction indicated by the progression of time, $t$. During the time $t_{\alpha}-t_{3}$, the time lag of the event seen at spacecraft $\alpha$ to that seen at the reference spacecraft (C3), the plane moves along the boundary normal a distance equal to the projection of the separation distance of the two spacecraft on to the plane normal:

$\left(\boldsymbol{r}_{\alpha}-\boldsymbol{r}_{3}\right) \cdot \hat{n}=V\left(t_{\alpha}-t_{3}\right)$,

where $\boldsymbol{r}$ is the position vector of the spacecraft from some origin. When all spacecraft are considered, the above is generalized to:

$D \frac{\hat{n}}{V}=T$,

where the matrix $D$ and linear array $T$ are:

$$
D=\left(\boldsymbol{r}_{1}-\boldsymbol{r}_{3}, \boldsymbol{r}_{2}-\boldsymbol{r}_{3}, \boldsymbol{r}_{4}-\boldsymbol{r}_{3}\right) \text { and } T=\left(\begin{array}{c}
t_{1}-t_{3} \\
t_{2}-t_{3} \\
t_{4}-t_{3}
\end{array}\right) \text {. }
$$



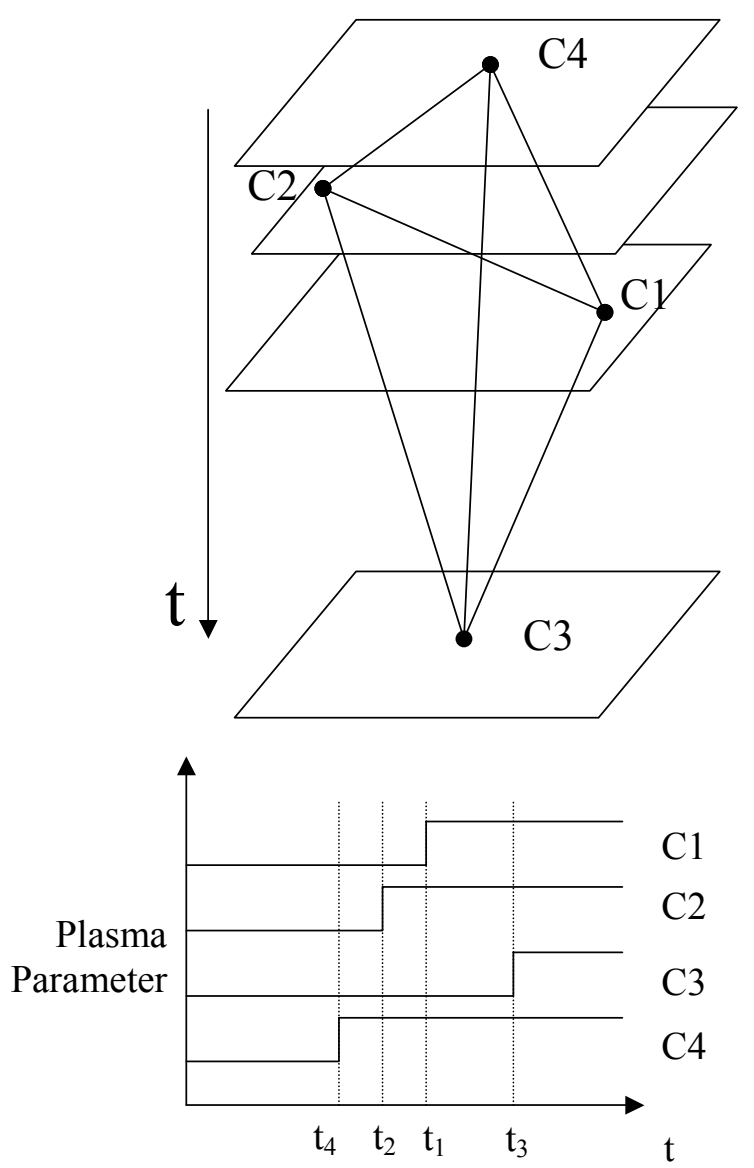

Fig. 1. A boundary between two distinct plasma regions passes over the Cluster quartet, marked by $\mathrm{C} 1$ to $\mathrm{C} 4$, and can be observed to cross spacecraft $\alpha$ at time $t_{\alpha}$. The plasma boundary is marked by a plane moving in the negative GSM-Z direction with time $t$. As the boundary passes each spacecraft for the different plasma populations a change is seen in the parameter time-series, shown here for all four spacecraft. The boundary is assumed to be planar on the scale of the tetrahedron.

If the spacecraft are not co-planar, then $D^{-1}$, the inverse of $D$, exists and the unit normal and the boundary velocity can be calculated:

$\frac{\hat{n}}{V}=D^{-1} T$.

In order to practically implement this method, we need to identify a plasma parameter that shows a gradient at the boundary of interest. For the lobe - plasma sheet interface there is usually a significant change in the plasma density. However, even the HEEA sensor, which for tail pass operations is usually set to detect electrons in the range $34 \mathrm{eV}$ to $\sim 26.5 \mathrm{keV}$, may at times be contaminated with photoelectrons from the spacecraft. This prevents the routine use of the onboard moments for our study. Hence, we construct on the ground a pseudo-density moment using the pitchangle distributions (PAD) that are routinely returned from the PEACE instrument at four-second resolution for the interval surrounding the spacecraft traversal of a lobe - plasma sheet interface. This data product consists of a slice from the full distribution containing 13 pitch-angle bins, selected on board by reference to the magnetometer data, which are each divided into 30 energy bins. This data product is available regardless of the telemetry mode.

We first identified for each period of interest the highest energy level bin that was contaminated with photoelectrons at some point within the period. We then perform a pseudodensity moment calculation from the pitch-angle distribution, assuming gyrotropy of the original distribution, and integrating only across those energy bins above this contaminated level. We thus form a rough proxy to the electron density in that energy range. This parameter can be used to make a clear determination of the time of the transition between plasma sheet and the lobe by each spacecraft.

We apply a cross-correlation technique to the resulting four pseudo-density data sets, in order to determine the relative time lags of the boundary crossing at each spacecraft. Given knowledge of the relative position of each spacecraft, Eq. (3) can then be used to calculate the normal and speed for an assumed planar boundary. As the pitch-angle distributions used in this method are obtained once per spacecraft spin, approximately $4 \mathrm{~s}$, there is up to a $\pm 2 \mathrm{~s}$ error on the timing of the crossing. This is the most significant source of error on the calculation of the boundary characteristics, as the error on spacecraft location is small compared to the spatial scales involved.

Note that the method used here assumes that the plasma sheet boundary is locally planar on the scale of the spacecraft separations. Thus, only the normal velocity, which is provided by this method, has any meaningful information. More global dynamics, such as large-scale transverse waves, cannot be inferred directly from any one velocity determination. We attempt in this paper to illuminate such dynamics by examining statistically the average motion of the edge of the plasma sheet as a function of position within the tail.

\section{Results}

To illustrate the various observations of plasma sheet thinning and expansion by the Cluster quartet, two case studies are presented. The first shows one isolated thinning and expansion cycle. The second example shows two cycles of plasma sheet thinning and expansion which occur during a comparatively more complicated substorm. Following the case studies we present various statistics from a survey of two years of magnetotail data.

\subsection{Case study: Single thinning and expansion}

An example of thinning and expansion of the plasma sheet over the Cluster quartet located at $\sim(-17.4,-5.5$, $-2.6)_{\mathrm{GSM}} \mathrm{R}_{E}$ is now presented. Ancillary data that indicates the occurrence of a substorm on 13 August 2001 is provided first as context for the Cluster data. 


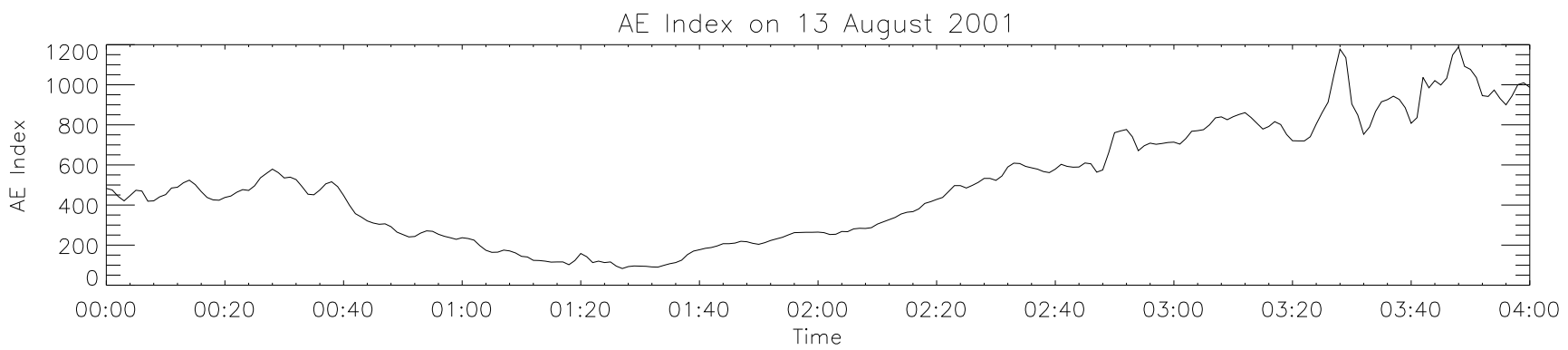

Fig. 2. The AE index (nT) for the period 00:00 UT to 04:00 UT on 13 August 2001. Of note is the intensification around 02:50 UT of $\sim 200 \mathrm{nT}$ which is associated with dynamics in the plasma sheet.

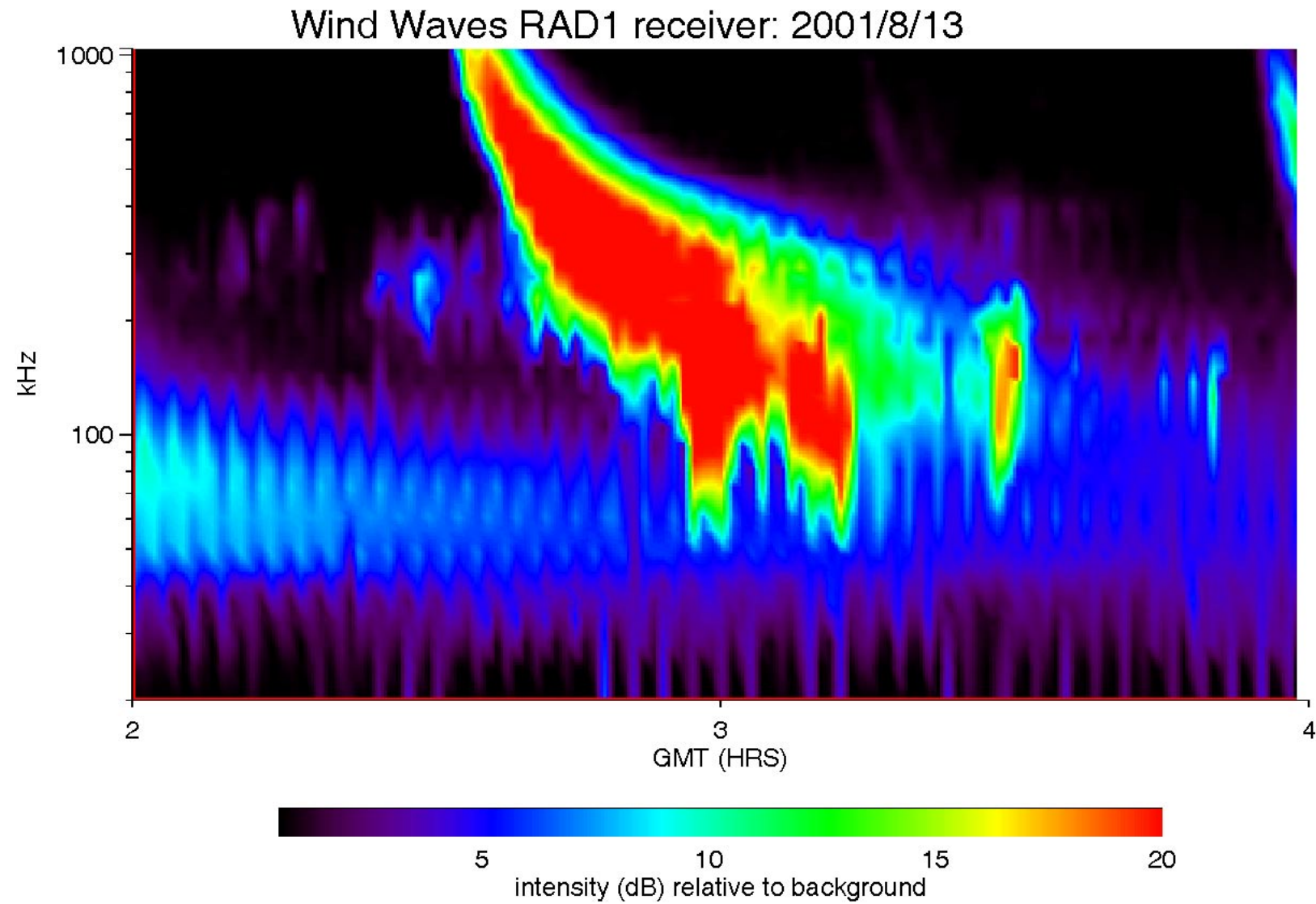

Fig. 3. A frequency spectrogram of the RAD1 receiver on the Waves experiment on board the Wind spacecraft for the period 02:00 UT to 04:00 UT on 13 August 2001. Onset of auroral kilometric radiation occurs at 02:55 UT with an enhancement of the intensity of the wave activity at $\sim 60$ to $100 \mathrm{kHz}$.

The AE trace shown in Fig. 2 shows that prior to $\sim 01: 30$ UT the system was in a recovery phase. A slow buildup of the AE index follows and is then punctuated by a rise of $\sim 200 \mathrm{nT}$ at approximately 02:50 UT, lasting $\sim 8 \mathrm{~min}$. This onset is followed by several others associated by much larger AE index values from 03:30 UT onwards.

A possible substorm onset time is given as 02:40 UT as defined by the presence of at least 2 min of continuous $\mathrm{Pi} 2 \mathrm{ac}-$ tivity after at least a 10 -min period of inactivity in the SAMNET magnetometer chain magnetic field $\mathrm{H}$-component data (not shown). The onset of a negative bay in Canopus ground magnetometer data occurs at 02:50 UT. An electron injection is seen on mid-energy channels of 1994-084's SOPA LoE at $\sim 02: 35$ UT and at LANL-01A at $\sim 03: 00$ UT (not shown). In Fig. 3 a frequency $(\mathrm{kHz})$ against time plot from Wind Waves (RAD1 detector) with the intensity $(\mathrm{dB})$ on the colour scale shows a Type III radio burst, with frequencies higher than $100 \mathrm{kHz}$, with auroral kilometric radiation superimposed at $\sim 02: 55$ UT. Geotail was not located in the magnetotail during this interval. 

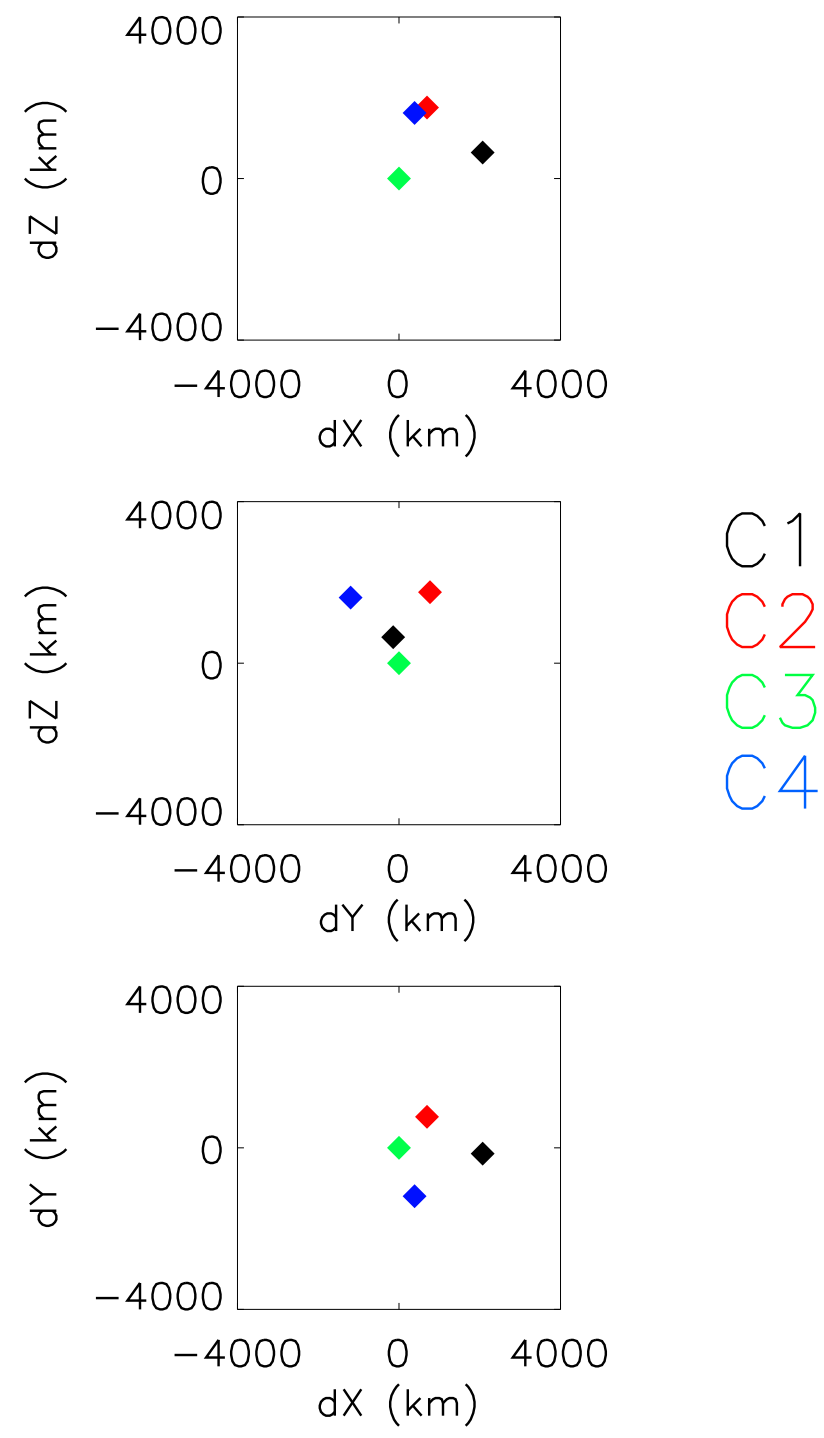

Fig. 4. The separations of each of the Cluster spacecraft $(\mathrm{C} 1, \mathrm{C} 2$ and $\mathrm{C} 4)$ from the reference spacecraft $(\mathrm{C} 3)$ position are shown in the GSM-XY, $-\mathrm{XZ}$ and $-\mathrm{YZ}$ planes. $\mathrm{C} 1, \mathrm{C} 2, \mathrm{C} 3$ and $\mathrm{C} 4$ are marked in black, red, green and blue, respectively. The order of the spacecraft in the GSM-Z direction is the expected order that the plasma sheet - lobe boundary should traverse the spacecraft quartet if the motion is purely in the GSM-Z direction.

An example of plasma sheet thinning and expansion observed by Cluster located at $\sim(-17.4,-5.5,-2.6)_{\mathrm{GSM}} \mathrm{R}_{E}$ is presented. The spacecraft configuration, showing the separation of the spacecraft from the reference position, is shown in Fig. 4 with $\mathrm{C} 1$ to 4 marked in black, red, green and blue, respectively. It should be noted that $\mathrm{C} 3$ is the furthest south of the four spacecraft, followed by $\mathrm{C} 1$, which is displaced in X from the other spacecraft, and then $\mathrm{C} 4$ and $\mathrm{C} 2$. This order should be thus similar to that of the detection by each spacecraft of a boundary that lay in the GSM-XY plane and travelled purely in the GSM-Z direction during the thinning and expansion phases.

Data from the PEACE instrument with ancillary magnetic field data is shown in Fig. 5. Panels 1 to 4 show omnidirectional energy spectrogram in differential energy flux of the electrons detected by the HEEA detector for each spacecraft, $\mathrm{C} 1$ down to $\mathrm{C} 4$, for a 50-min interval, 02:10 to 03:00 UT. Electron energy is split into 30 bins between $34 \mathrm{eV}$ and $\sim 26.5 \mathrm{keV}$ and the differential energy flux scale runs between $10^{-6}$ and $10^{-4}$ ergs $\left(\mathrm{cm}^{2} \mathrm{~s} \text { str ev }\right)^{-1}$. Note that in the south lobe (between $\sim 02: 23$ UT and $\sim 02: 50$ UT) each spacecraft observes significant fluxes of photoelectrons within the HEEA energy range which reach energies of up to $\sim 100 \mathrm{eV}$ (and at $\sim 02: 46$ UT on spacecraft 2 there are signatures associated with the operation of the Electron Drift Instrument). These photoelectrons dominate the onboard moments (not shown) when the spacecraft are in the lobe, and thus we use the pseudo-moment shown in panel 5 for our analysis (black, red, green and blue for spacecraft number 1 , 2, 3 and 4, respectively). This data was calculated using the method described above taking all energy bins above, $135 \mathrm{eV}$ on all spacecraft to ensure the comparison of the similar data products. The magnetic field $\mathrm{B}_{x}$ and $\mathrm{B}_{z}$ components in GSM co-ordinates are then shown in panels 6 and 7. Data from each of the four spacecraft are shown, with $\mathrm{C} 1$ data represented by the black trace, $\mathrm{C} 2$ by red, $\mathrm{C} 3$ by green and $\mathrm{C} 4$ by blue.

At the beginning of the period shown, each spacecraft is in a low-energy plasma sheet, with an electron distribution centred on $\sim 250 \mathrm{eV}$. C1, C2, C3 and C4 moved out of the plasma sheet into the south lobe at $\sim 02: 21: 23 \mathrm{UT}, 02: 20: 53 \mathrm{UT}$, 02:21:14 UT and 02:22:03 UT, respectively, i.e. in the order, C2, C3, C1 and C4. Although we might have expected that a transition from the plasma sheet to the south lobe be associated with a boundary lying the GSM-XY plane moving predominately northward, in this case application of the timing analysis reveals that the boundary lies almost entirely in the GSM-XZ plane and moves in the dawnward direction along the unit normal $\sim(0.06,-0.96,0.27)_{\mathrm{GSM}}$ with a speed along that normal of $\sim 23 \mathrm{~km} \mathrm{~s}^{-1}$. The four spacecraft re-enter the plasma sheet with the lobe - plasma sheet boundary observed at 02:50:05 UT, 02:49:41 UT, 02:50:30 UT and 02:49:47 UT at C1, C2, C3 and C4, respectively, i.e. in the order $\mathrm{C} 2, \mathrm{C} 4, \mathrm{C} 1$ and $\mathrm{C} 3$. This corresponds to the boundary moving at $\sim 49 \mathrm{~km} \mathrm{~s}^{-1}$ along the unit normal $\sim(0.02,-0.23,-0.97)_{\mathrm{GSM}}$, indicating a boundary lying in the GSM-XY plane which moves almost entirely in the negative GSM-Z direction, as we might naïvely have expected for a transition from the south lobe into the plasma sheet. Note also that the spacecraft now enter a plasma sheet which is hotter than that observed previously, with the distribution centred at $\sim 1 \mathrm{keV}$.

The magnetic field at each spacecraft, shown in Fig. 5, panels 6 and 7, indicates a clear example of a major reorganisation of the magnetic field during this interval. At $\sim 02: 51$ UT the magnetic field in the GSM-X direction is reduced by approximately $20 \mathrm{nT}$ and is accompanied by a strengthening of the GSM-Z component of the magnetic field by approximately $10 \mathrm{nT}$. This indicates a magnetic field dipolarization. 

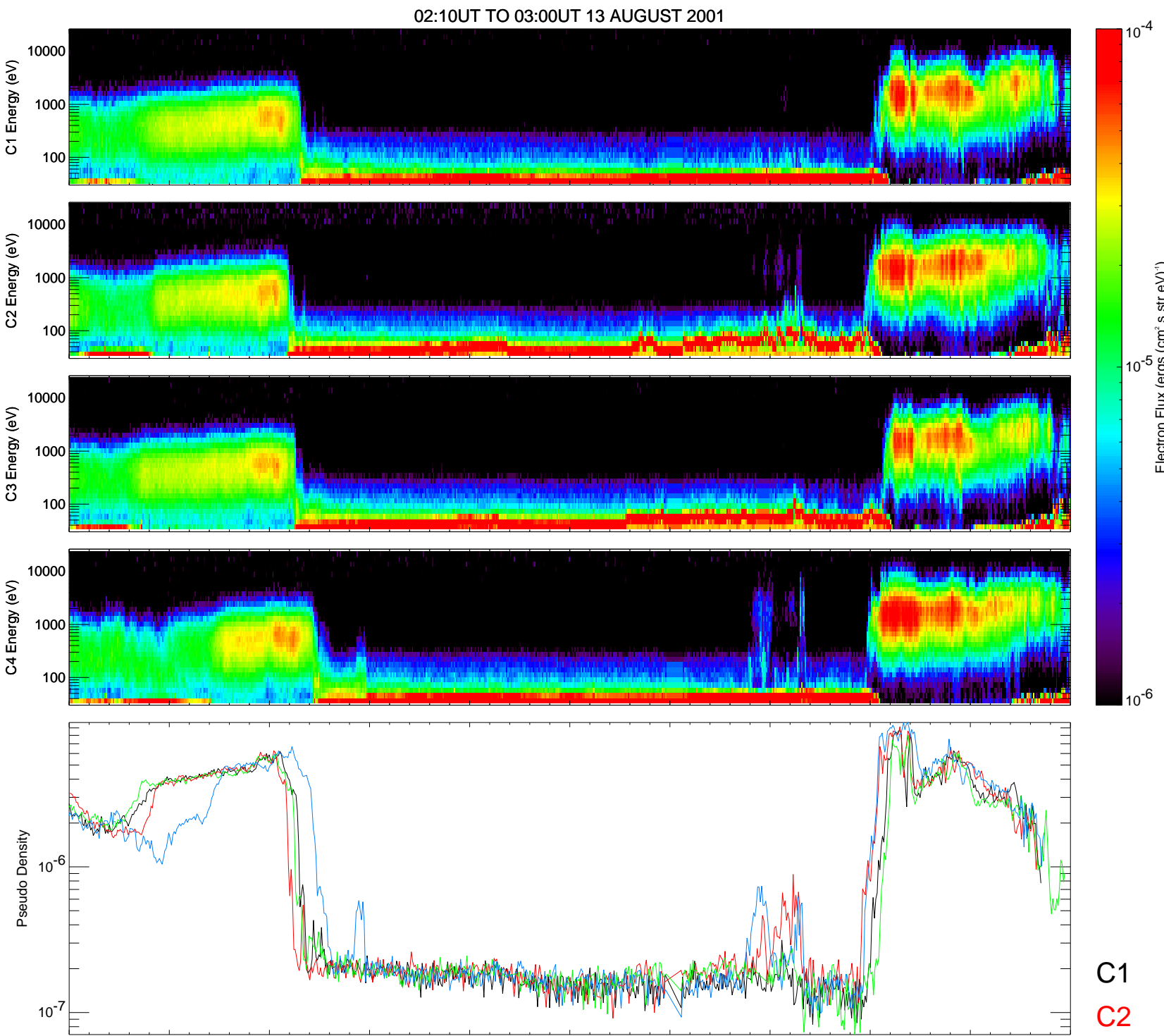

$\mathrm{C} 1$
$\mathrm{C} 2$
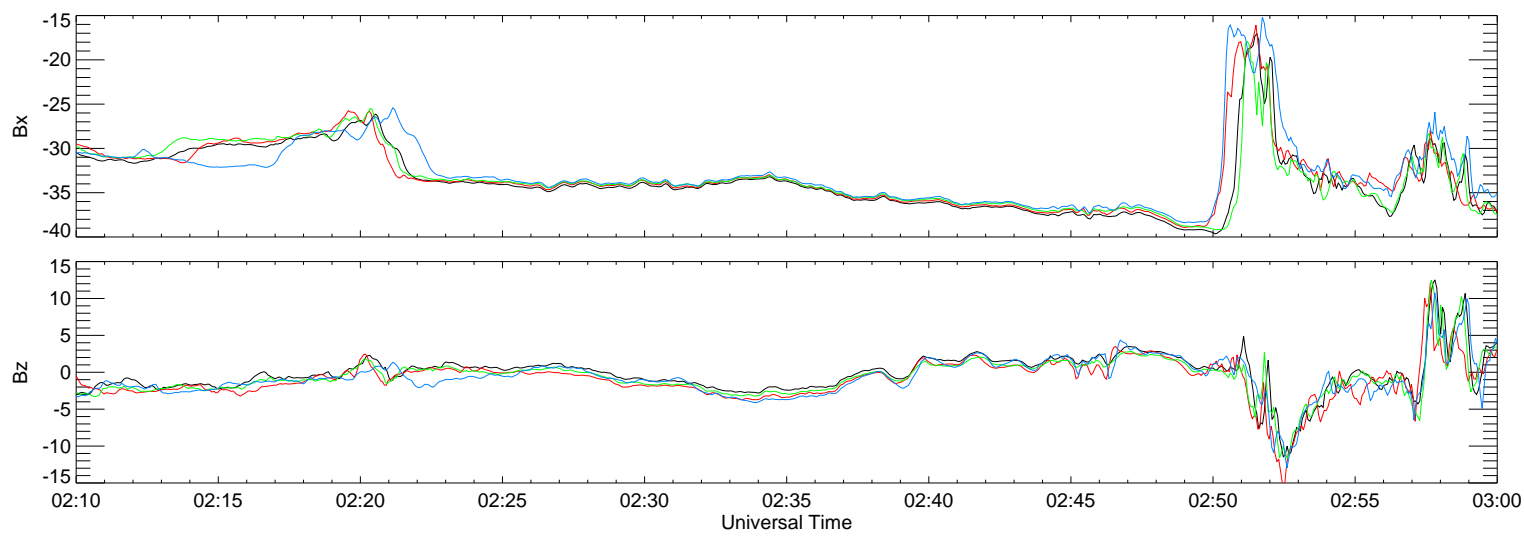

Fig. 5. Electron energy - differential flux spectrogram for $\mathrm{C} 1$ to $\mathrm{C} 4$ in the first four panels showing data summed over all pitch angles for the interval 02:10 UT to 03:00 UT on 13 August 2001. Plasma sheet plasma is punctuated by a period in the lobe. A density proxy (summed of all electrons above $135 \mathrm{eV}$ ) is plotted in panel 5 using black, red, green and blue for $\mathrm{C} 1$ to $\mathrm{C} 4$, respectively. The $\mathrm{B}_{X}$ and $\mathrm{B}_{Z}$ components of the magnetic field measured at $\mathrm{C} 3$ are shown in panels 6 and 7 . A clear magnetic field rearrangement is seen at $\mathrm{C} 3$ around the time of the plasma sheet passing over the spacecraft quartet. 

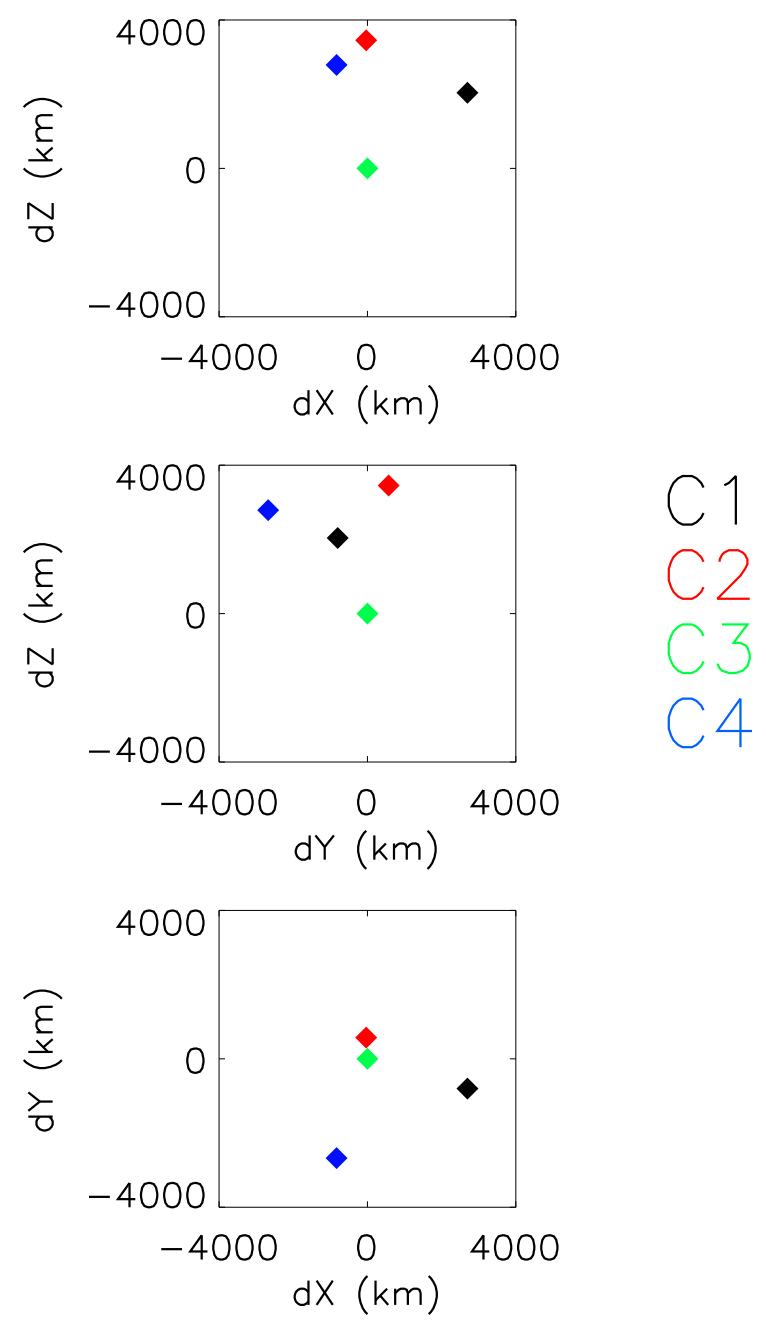

Fig. 6. The separations of each of the Cluster spacecraft from the reference position are shown in the GSM-XY, $-\mathrm{XZ}$ and $-\mathrm{YZ}$ planes. $\mathrm{C} 1$ to $\mathrm{C} 4$ are marked in black, red, green and blue respectively. The order of the spacecraft in the GSM-Z direction is the expected order the plasma sheet - lobe boundary should traverse the spacecraft quartet.

The thinning of the plasma sheet at Cluster is seen at $\sim 02: 21$ UT. Pi2 pulsations observed at SAMNET at $\sim 02: 40$ UT preceded events in both the AE index and Canopus magnetometer data which place the onset of the substorm at $\sim 02: 50 \mathrm{UT}$, which may by explained by the differences in local time of the various observations. At this time the plasma sheet is seen to expand over the Cluster quartet and the onset of auroral kilometric radiation is seen shortly after at $\sim 02: 55$ UT. At 03:00 UT an injection is seen at geosynchronous orbit.

\subsection{Case study: Multiple thinning and expansion}

There are two instances of multiple plasma sheet thinning and expansion associated with complex geomagnetic activity within our data set. Analysis may provide a measure of how much the plasma sheet changes throughout the course of a complex substorm. For example, changes in the orientation of the neutral sheet may occur and therefore affect the direction of future thinnings and expansions.

To illustrate this, we take the repeated thinnings and expansions of the plasma sheet that were observed by Cluster on 11 August 2002 between 16:40 UT and 16:54 UT when Cluster was located at $(-17.6,-7.1,2.0)_{\mathrm{GSM}} \mathrm{R}_{E}$. The $\mathrm{AE}$ index is not available in digital form for this period and a description of the data follows. Prior to this interval, the AE index indicates two large substorm onsets at $\sim 14: 15$ UT and $\sim 14: 35$ UT with AE index values of $\sim 750 \mathrm{nT}$ and $\sim 1200 \mathrm{nT}$, respectively. The $\mathrm{AE}$ at the beginning of this study interval had reached $\sim 700 \mathrm{nT}$, after rising steadily from $\sim 550 \mathrm{nT}$ at $\sim 16: 25$ UT. At $\sim 16: 50$ UT there is an enhancement of the $\mathrm{AE}$ index to $\sim 800 \mathrm{nT}$, returning to $\sim 700 \mathrm{nT}$ before an enhancement to $\sim 750 \mathrm{nT}$ at 17:00 UT. A chain of intensifications to near 500 nT follow 18:00 UT. A slight enhancement of proton differential flux as detected by SOPA LoP (not shown) on LANL-02A and at LANL-97A at geosynchronous orbit at $\sim 16: 35 \mathrm{UT}$, with similar signatures seen in the SOPA LoE electron data (not shown). Geotail was not located in the magnetotail during this interval.

The position of each of the spacecraft is shown in Fig. 6 using the same format as Fig. 4. Note that Cluster 1 is separated in GSM-X from the other spacecraft. Data from Cluster PEACE and the magnetometer for the interval 16:40 to 16:54 UT are shown in Fig. 7, with the same structure as that of Fig. 5. Prior to the start of the interval shown in this figure, Cluster had been located in the plasma sheet since $\sim$ 14:47 UT, observing varying electron distributions centred between 2 to $6 \mathrm{keV}$. At the beginning of the period shown in Fig. 7, each spacecraft is in a high-energy plasma sheet with an electron distribution centred on $\sim 2 \mathrm{keV}$. This is a significantly higher energy electron population when compared to the example in Fig. 5, and is probably due to the heating that occurred from the previous extensive substorm activity. The application of the methodology described in Sect. 2 indicated that $\mathrm{C} 1, \mathrm{C} 2, \mathrm{C} 3$ and $\mathrm{C} 4$ moved out of the plasma sheet into the south lobe at $~ 16: 43: 20 \mathrm{UT}, 16: 43: 47 \mathrm{UT}, 16: 42: 45 \mathrm{UT}$ and 16:43:38 UT, respectively, i.e. in the order C3, C1, C4 and $\mathrm{C} 2$. The quartet returned to the plasma sheet, now with an electron population distribution centred on $3 \mathrm{keV}$ and a higher energy flux, at 16:46:12 UT, 16:45:39 UT, 16:46:46 UT and 16:45:22 UT for Clusters 1 to 4, respectively, i.e. in the order $\mathrm{C} 4, \mathrm{C} 2, \mathrm{C} 1$ and $\mathrm{C} 3$. The thinning of the plasma sheet occurred again at $\mathrm{C} 1, \mathrm{C} 2, \mathrm{C} 3$ and $\mathrm{C} 4$ at $\sim 16: 49: 20 \mathrm{UT}, 16: 48: 59 \mathrm{UT}, 16: 48: 18$ and 16:49:39 UT (i.e. C3, C2, C4 then C1) and returned at 16:50:12 UT, 16:49:55 UT, 16:51:15 UT and 16:50:11 UT (i.e. C2, C4, $\mathrm{C} 1$ then $\mathrm{C} 3$ ) to a similarly energised plasma sheet.

The first entry into the south lobe is modelled by a planar boundary moving along a unit normal largely in positive GSM-Z of $\sim(0.03,0.31,0.95)_{\mathrm{GSM}}$ with a speed of $35 \mathrm{~km} \mathrm{~s}^{-1}$. The analysis of the subsequent expansion of the plasma sheet returns a unit normal $\sim(-0.02,-0.66$, $-0.75)_{\mathrm{GSM}}$ and a speed of $38 \mathrm{~km} \mathrm{~s}^{-1}$ along that normal. The second thinning has a plane of unit normal in GSM- 

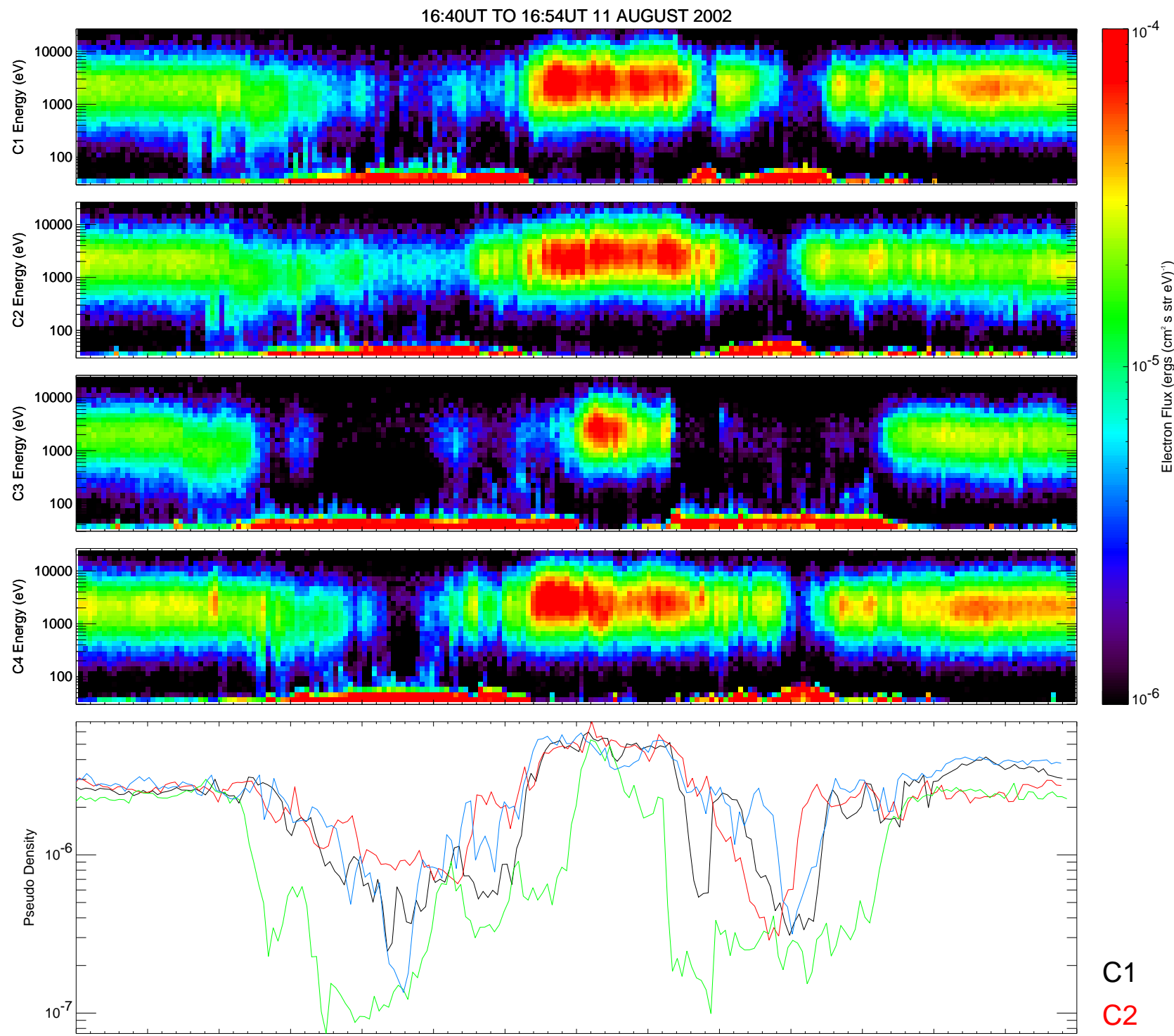

C1

C2

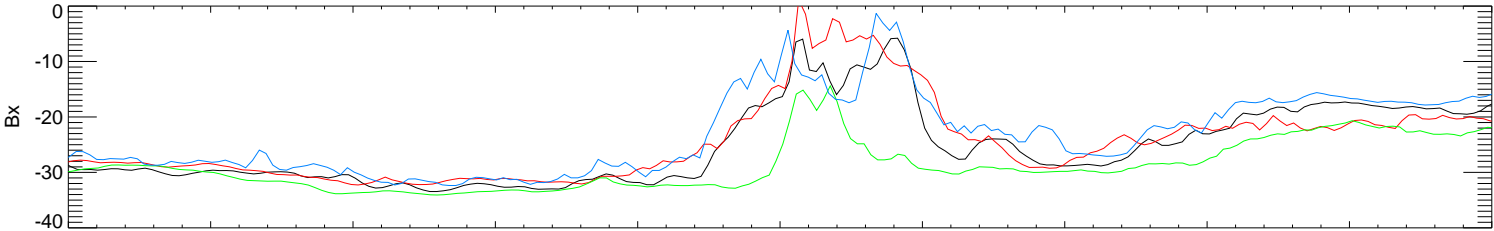

C3

C4

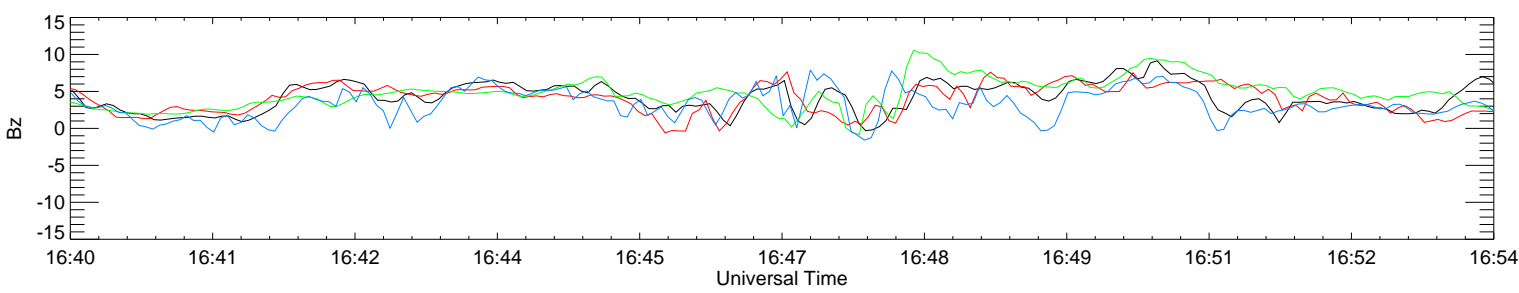

Fig. 7. Electron energy - differential flux spectrogram for $\mathrm{C} 1$ to $\mathrm{C} 4$ in the first four panels showing data summed over all pitch angles for the interval 16:40 UT to 16:54 UT on 11 August 2002. Plasma sheet plasma is punctuated by two periods in the lobe. The density proxy (summed over all electrons above $\sim 100 \mathrm{eV}$ ) is plotted in panel 5 using black, red, green and blue for $\mathrm{C} 1$ to $\mathrm{C} 4$, respectively. The $\mathrm{B}_{X}$ and $\mathrm{B}_{Z}$ components of the magnetic field measured at C3 are shown in panels 6 and 7, showing a magnetic field re-arrangement at $\sim 16: 47$ UT. 

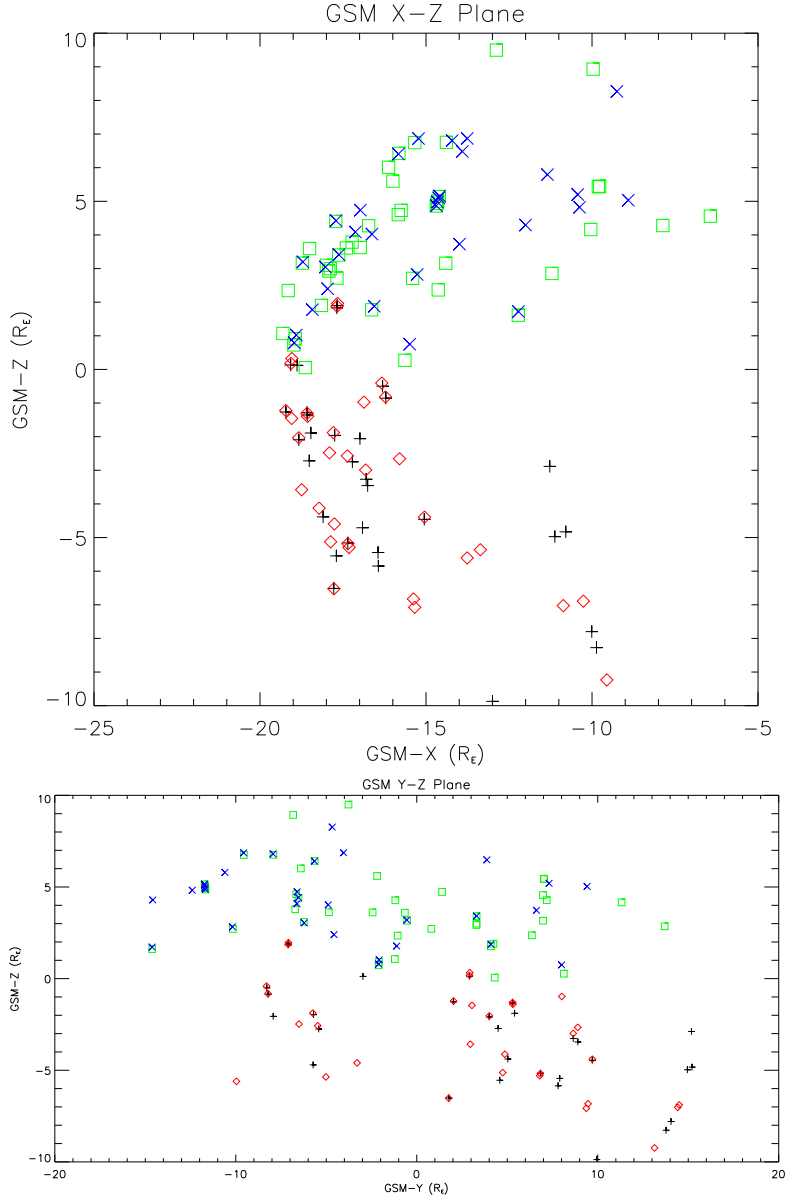

Fig. 8. The distribution of all the passages of the spacecraft from the lobe into the plasma sheet and vice versa in the GSM-YZ and GSM-XY planes. Expansion crossing are marked by green squares and black plus signs for the north and south lobes, respectively. Thinning crossings are indicated by blue crosses and red diamonds in the north and south lobes, respectively. The data points are evenly distributed over the GSM-YZ plane.

$\mathrm{X}$ and $\mathrm{GSM}-\mathrm{Z} \sim(0.84,0.19,0.51)_{\mathrm{GSM}}$ with a speed of $48 \mathrm{~km} \mathrm{~s}^{-1}$, with a subsequently associated expansion with a plane unit normal again in negative GSM-Z $\sim(-0.21$, $-0.45,-0.87)_{\mathrm{GSM}}$ and a speed of $45 \mathrm{~km} \mathrm{~s}^{-1}$ along that normal. The GSM-Z components provide further evidence that the expansion vectors follow the expected direction while the thinning vectors are more varied in this case. In these two thinnings the prominent direction for the thinning is the GSM-X direction. It may have been expected that the second event had less energy for a vigorous expansion, but the speeds of thinning and expansion were greater and in approximately the same ratio as the first paired event. However, the complexity of substorms with multiple onsets and the lack of observations of thinnings and expansions associated with each onset of a complex substorm provide interpretational difficulties.

The magnetic field from all spacecraft, using the same colour code as above, is shown in Fig. 6, panels 6 and 7 , and indicates a reorganisation of the magnetic field at $\sim 16: 47: 10$ UT and possibly one at $\sim 16: 51$ UT. Whilst there is a reduction of approximately $15 \mathrm{nT}$ in $\mathrm{B}_{X}$ only slight variation occurs in $B_{Z}$. These field changes may be due to total pressure balance between low to high $\beta$ plasma.

Two tail seasons of Cluster data were available for this study. In the following section survey statistics of the observed substorm-associated plasma sheet thinnings and expansions will be described.

\subsection{Statistical survey}

We surveyed two tail seasons to identify all substorm-related expansions and contractions of the plasma sheet observed at the Cluster spacecraft. To distinguish between substormrelated activity (both growth phase related thinning and onset related expansion) and the passage of the plasma sheet over the spacecraft related to diurnal motion and boundary waves, only crossings that are located within $15 \mathrm{~min}$ of an $\mathrm{AE}$ intensification (defined here as an increase of at least $50 \mathrm{nT}$ ), where the background $\mathrm{AE}$ is $\mathrm{AE} \geq 100 \mathrm{nT}$, were selected. No estimate of the substorm onset time was made due to data availability constraints and the known difficulty in performing this type of analysis (c.f. Liou, 1999). In addition, boundaries with normal velocities on the order of that of the spacecraft orbital motion $\left(\sim 3 \mathrm{~km} \mathrm{~s}^{-1}\right)$ are not included in our database. These are probably not substorm-related and are subject to significant errors, as it is unlikely that the boundary has remained both planar and moved at a constant velocity throughout the associated extended time lag between spacecraft. A total of 44 passages from the north lobe to the plasma sheet were observed and 33 transitions were observed from the south lobe to the plasma sheet, corresponding to plasma sheet expansion. In addition, there were 31 passages from the plasma sheet to the north lobe, and 35 to the south lobe corresponding to plasma sheet thinnings.

The distribution of events satisfying the above criteria is shown in Fig. 8 in the GSM co-ordinate system. The GSM$\mathrm{XZ}$ plane is shown in the top panel with the GSM-X axis shown from $-5 \mathrm{R}_{E}$ to $-25 \mathrm{R}_{E}$ and the GSM-Z axis shown from -10 to $10 \mathrm{R}_{E}$. The GSM-YZ plane is shown in the bottom panel with the GSM-Y axis plotted from -20 to 20 in Earth Radii, $\mathrm{R}_{E}$, and with the GSM-Z axis again shown from -10 to $10 \mathrm{R}_{E}$. The positions of the 44 passages from the north lobe to the plasma sheet and 33 transitions from the south lobe to the plasma sheet are represented by green squares $(\square)$ and black plus signs (+), respectively, in the plots. The positions of the 31 passages from the plasma sheet to the north lobe and 35 to the south lobe are represented by blue crosses $(\times)$ and red diamonds $(\diamond)$, respectively. The GSM-YZ plane clearly shows that substorm associated lobe - plasma sheet boundary motions were detected across the entire magnetotail from dawn to dusk. Groups of events, for example, that at $\sim(-12.5,5)_{\mathrm{GSM}} \mathrm{R}_{E}$ in the GSM-YZ plane, occur during very complex substorm activity, there are repeated plasma sheet thinnings and expansions within only a 
few hours and thus only a small change of spacecraft position occurred. There are 29 pairs of events (c.f. case 1 above) where the spacecraft goes from the plasma sheet into the lobe and then back again. However, it should be also noted that there are often missing "partner" events where the data are too complex for boundary analysis (e.g. on one or more spacecraft there are several possible points that could be taken as the plasma sheet - lobe boundary that corresponds to the boundary observed on other spacecraft, or one or more spacecraft did not cross the boundary) or instrument operation ceases on one or more spacecraft prior to the partner event.

We now move on to consider the results of the timing analysis of the events in this survey. We first consider events associated with the apparent thinning of the plasma sheet, i.e. those in which the spacecraft moves from the plasma sheet into the lobe. Figure 9 shows the same GSM-XZ and -YZ planes as those used in Fig. 8. The location of the 31 passages from the plasma sheet to the north lobe and the 35 passages from the plasma sheet to the south lobe are again represented by blue crosses $(\times)$ and red diamonds $(\diamond)$, respectively. In addition, for each thinning the appropriate projection of the unit normal of the lobe - plasma sheet boundary for that event is shown. A unit normal within each of the planes is shown for guidance. The possible error in direction of this normal vector arises from the \pm 2 s error on the timing, indicated by dotted lines. The size of the error on the normal measurement is associated with the speed of the boundary over the spacecraft quartet: when the boundary crosses some or all of the spacecraft within $\sim 4 \mathrm{~s}$ the normal vector could be completely incorrectly determined. Naturally, large delays of minutes introduce errors in the assumptions made, as it is impossible to say exactly how the boundary travels between observations. Situations when delays of several minutes exist have not been included and they are likely to not be substorm-associated. In the Northern Hemisphere the normal vector (blue vectors) in the GSM-YZ plane usually points towards the expected location of the current sheet, although there are notable exceptions towards high GSM-Z values where they tend to have mostly a GSM-Y component. In the Southern Hemisphere (red vectors) this trend is replaced by a mainly negative GSM-Y component of the normal. In the GSM-XZ plane there is no clear overall trend in the GSM-X component of the unit normal. We now bin the data into smaller distance ranges to allow further clarity.

The data were binned into six $6 \mathrm{R}_{E}$ bins along the GSM$\mathrm{Y}$ axis and into four $5 \mathrm{R}_{E}$ bins in the GSM-Z axis for the study of the GSM-YZ vector of the thinning of the plasma sheet in four $4 \mathrm{R}_{E}$ bins along the GSM-X axis. For the study of the GSM-XZ vector of the thinning, the data were binned into four $4 \mathrm{R}_{E}$ bins along the GSM-X axis and six $6 \mathrm{R}_{E}$ bins along the GSM-Y axis summed over all events within the relevant lobe. Within each bin, the average normal direction for the thinnings in each hemisphere was calculated. There are data bins that suffer from low-frequency statistical errors, but binning into variable bin sizes would mask the details of the overview.
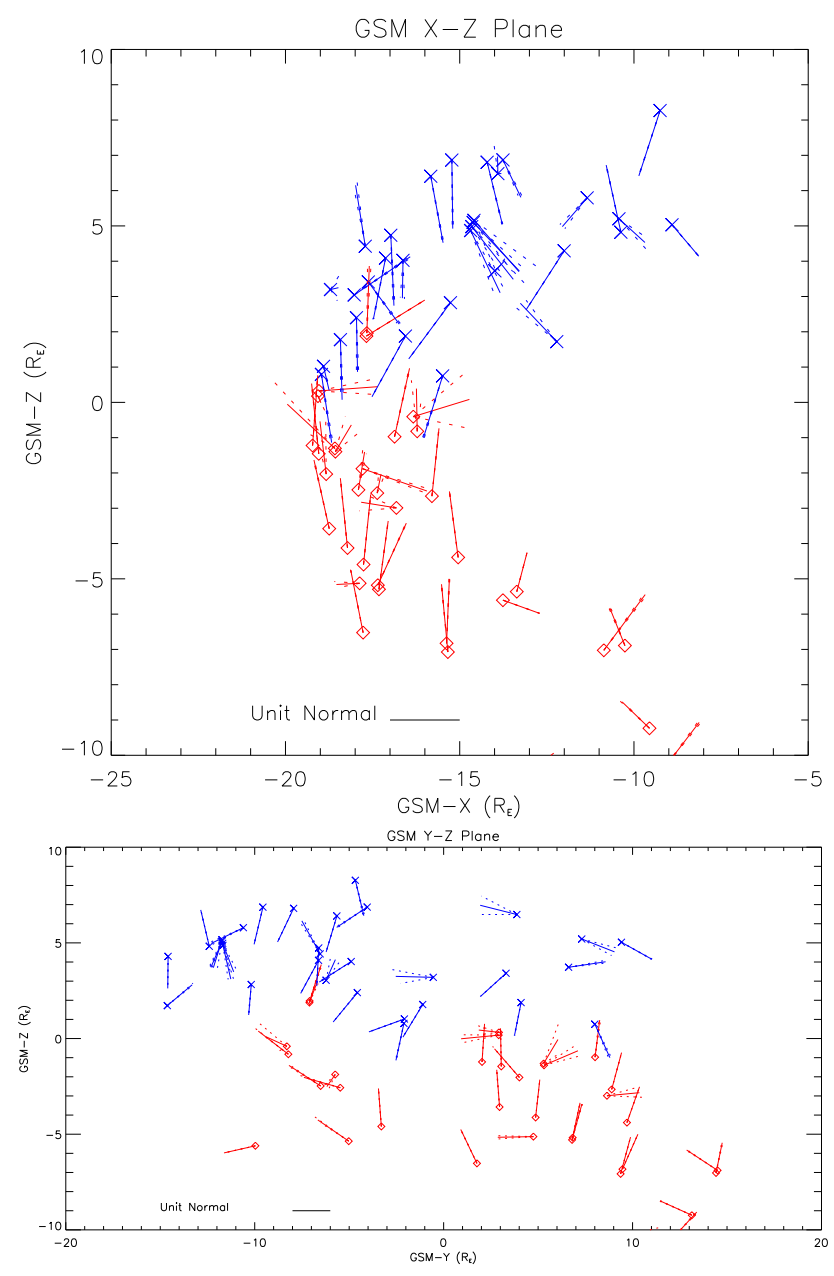

Fig. 9. For each passage of the spacecraft from the plasma sheet into the lobe on the GSM-YZ and GSM-XY planes, the projection of the lobe - plasma sheet boundary normal vector on that plane is shown, together with an estimate of the error associated with the spacecraft spin (dotted line). Those events in the Northern Hemisphere are shown in blue and those in the Southern Hemisphere are shown in red. A unit normal entirely in that plane is shown for reference. In the Northern Hemisphere there is a tendency for the vectors to point towards the expected location of the current sheet, although there are notable exceptions where the GSM-Y component of the boundary normal is dominant. In the south lobe there is a less clear trend for the vectors to point to the current sheet, instead the vectors contain a mainly negative GSM-Y component.

Figure 10 shows the GSM-YZ projection of the binned normal vector averages as a function of spacecraft position in the GSM-YZ plane for two of the four bins along the GSM$\mathrm{X}$ axis $\left(-20 \leq \mathrm{X}_{\mathrm{GSM}} \leq-16 \mathrm{R}_{E}\right.$ and $\left.-16 \leq \mathrm{X}_{\mathrm{GSM}} \leq-12 \mathrm{R}_{E}\right)$ for those boundaries associated with the spacecraft passing from the plasma sheet into the lobe. Each panel, labelled by GSM-X bin, has the GSM-Z bin along the ordinate axis against the GSM-Y bin along the abscissa. At each mesh point the projection of the average normal vector in the GSM-ZY plane of the plasma sheet - lobe boundary associ- 

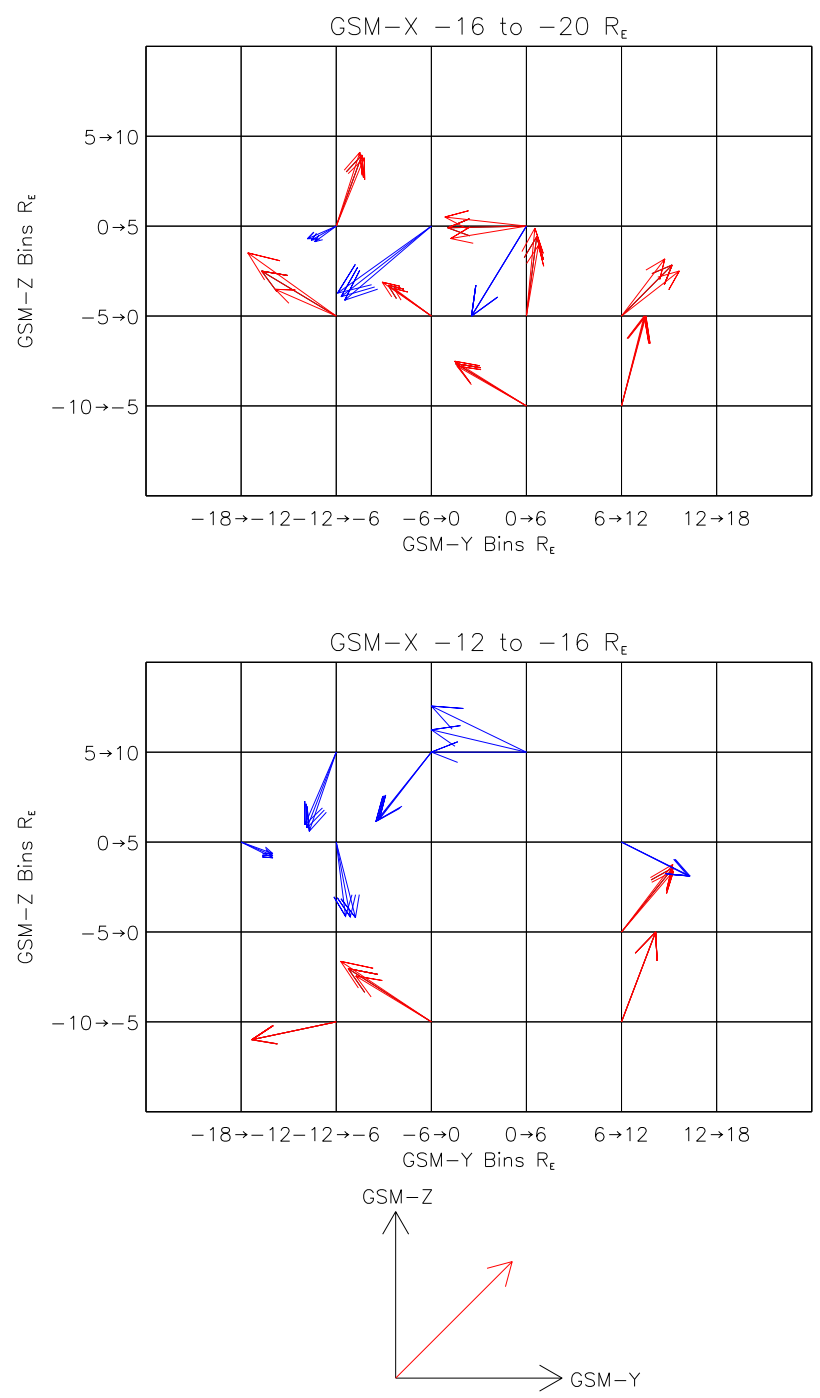

Fig. 10. The projection of the average unit normal of the boundary between the thinning plasma sheet and the lobe in the GSM-ZY direction is shown as a function of position in the GSM-ZY plane for two GSM-X bins. Plasma sheet to lobe crossings are marked in blue and red vectors for the north and south lobes, respectively. Error bars, calculated from the extreme errors on the individual vectors, are plotted when more than one normal vector was used for the averaging. The top panel shows that thinning in the north and south lobes tends to have a negative GSM-Y component with a variety of GSM-Z dominance. The bottom plot suggests that there is a greater variety of GSM-Y components closer to the Earth.

ated with plasma sheet thinning is shown for that bin. Events located in the north lobe are marked in blue and those in the south lobe marked in red. At each mesh-point the projection of the average normal vector created by taking the extreme timing error on the component normals is also shown to provide an assessment of the error. Due to the natural orbit procession and the inclination of the orbit, we expect there to be more data points in the central parts of the GSM-YZ plane for the $-20 \leq \mathrm{X}_{\mathrm{GSM}} \leq-16 \mathrm{R}_{E}$ bin for apogee and more points on the flanks for the $-16 \leq \mathrm{X}_{\mathrm{GSM}} \leq-12 \mathrm{R}_{E}$ bin.
The $-20 \leq \mathrm{X}_{\mathrm{GSM}} \leq-16 \mathrm{R}_{E}$ bin shows that the thinnings in the north and south lobes tend to have a negative GSM-Y component at all cross tail distances. All vectors (except $0 \leq \mathrm{Y}_{\mathrm{GSM}} \leq 6 \mathrm{R}_{E}$ and $0 \leq \mathrm{Z}_{\mathrm{GSM}} \leq 5 \mathrm{R}_{E}$ ) in this $\mathrm{X}_{\mathrm{GSM}}$ bin point towards the expected location of the neutral sheet. It is noted that the dominance of the GSM-Z component varies over the binned data, with the most predominantly GSM-Z thinning vectors being located in the positive GSM-Y part of the data set, except for the $0 \leq \mathrm{Z}_{\mathrm{GSM}} \leq 5 \mathrm{R}_{E}$ and $0 \leq \mathrm{Y}_{\mathrm{GSM}} \leq 6 \mathrm{R}_{E}$ bin. All the thinnings that occur in the north lobe (blue vectors) have a negative GSM-Z component, where all have a negative GSM-Y component.

The thinning averages for north lobe (blue vectors) in the $-16 \leq \mathrm{X}_{\mathrm{GSM}} \leq-12 \mathrm{R}_{E}$ bin show there is a tendency for the vectors to have a negative GSM-Z component, although the exception at $0 \leq \mathrm{Y}_{\mathrm{GSM}} \leq 6 \mathrm{R}_{E}$ and $5 \leq \mathrm{Z}_{\mathrm{GSM}} \leq 10 \mathrm{R}_{E}$ bin has a positive GSM-Z component. There is a greater variety in the direction of the GSM-Y component with the vectors pointing in the positive GSM-Y direction in the $0 \leq \mathrm{Z}_{\mathrm{GSM}} \leq 5 \mathrm{R}_{E}$ bin and then in the negative GSM-Z direction at higher GSM$\mathrm{Z}$ values. In the south lobe there is a thinning strongly towards the neutral sheet except at $\left(-16 \leq \mathrm{X}_{\mathrm{GSM}} \leq-12 \mathrm{R}_{E}\right.$ and $\left.-10 \leq \mathrm{Z}_{\mathrm{GSM}} \leq-5 \mathrm{R}_{E}\right)$. The thinnings have negative GSM-Y components in the post-midnight sector and positive GSM-Y components in the pre-midnight sector.

Figure 11 shows the GSM-XZ projection of the binned normal vector averages as a function of spacecraft position in the GSM-XY plane for the boundaries associated with the spacecraft passing from the plasma sheet into the lobe in each lobe. The panel has the GSM-Y bin along the ordinate axis against the GSM-X bin along the abscissa. At each mesh point the projection of the average normal vector in the GSM-XZ plane of the plasma sheet - lobe boundary associated with plasma sheet thinning is shown for that bin. Events located in the north lobe are marked in blue and those in the south lobe marked in red. At each mesh-point the projection of the average normal vector created by taking the extreme timing error on the component normals is again shown to provide an assessment of the error.

All but two of the thinning vectors in the south lobe (red vectors) have a positive GSM-Z component with the exceptions having a weak GSM-Z component $\left(-6 \leq \mathrm{Y}_{\mathrm{GSM}} \leq 0 \mathrm{R}_{E}\right.$ and $-16 \leq \mathrm{X}_{\mathrm{GSM}} \leq-12 \mathrm{R}_{E}$ ) and a strongly negative one $\left(6 \leq \mathrm{Y}_{\mathrm{GSM}} \leq 12 \mathrm{R}_{E}\right.$ and $\left.-12 \leq \mathrm{X}_{\mathrm{GSM}} \leq-8 \mathrm{R}_{E}\right)$. Considering the $-20 \leq \mathrm{X}_{\mathrm{GSM}} \leq-16 \mathrm{R}_{E}$ bin the thinning vectors in the dusk sector have only a slight GSM-X component and those in the dawn sector have a positive GSM-X component.

In the north lobe, the thinning vectors (blue vectors) all have a strongly to weakly negative GSM-Z component, except for the flank-most GSM-Y bin and the $0 \leq \mathrm{Y}_{\mathrm{GSM}} \leq 6 \mathrm{R}_{E}$ and $-16 \leq \mathrm{X}_{\mathrm{GSM}} \leq-12 \mathrm{R}_{E}$ bin which was noted as an exception in Fig. 10. There is no significant GSM-X component at any point along $\mathrm{GSM}-\mathrm{Y}$ in the $-20 \leq \mathrm{X}_{\mathrm{GSM}} \leq-16 \mathrm{R}_{E}$ bin with the strongest GSM-X components shown in the dawn sector within GSM-X $=-16 \mathrm{R}_{E}$.

We now go on to consider events that are associated with the expansion of the plasma sheet, i.e. those in which the 


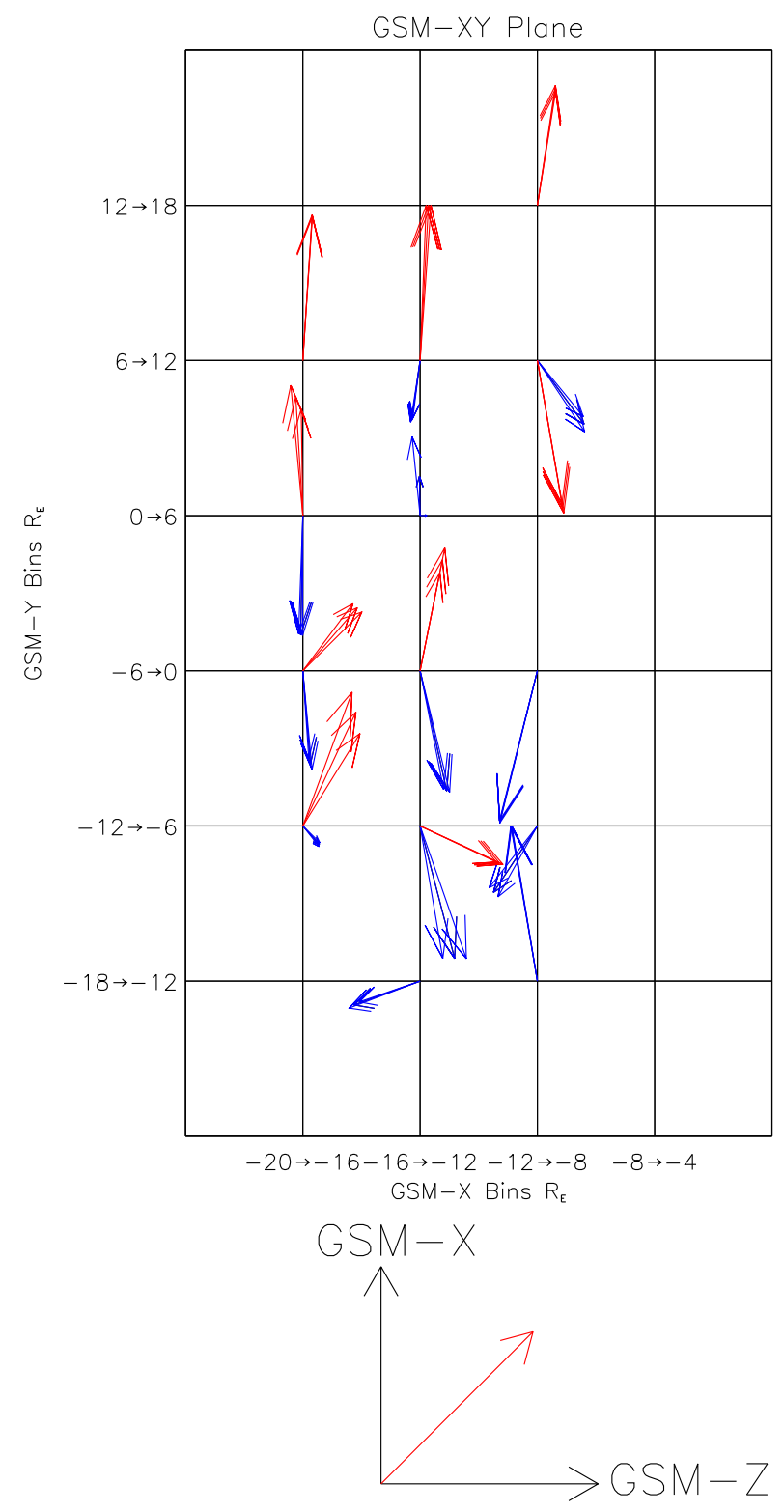

Fig. 11. The projection of the average unit normal of the boundary between the thinning plasma sheet and the lobe in the GSM-ZX direction is shown as a function of position in the GSM-YX plane for the north lobe (blue vectors) and south lobe (red vectors). Error bars, calculated from the extreme errors on the individual vectors, are plotted when more than one normal vector was used for the averaging.

spacecraft moves from the lobe into the plasma sheet. Figure 12 shows the projection of the unit normal of the lobe plasma sheet boundary for these events in the GSM-XZ and -YZ planes and has the same format as Fig. 9. The location of the 44 passages from the north lobe to the plasma sheet and the 33 passages from the south lobe to the plasma sheet are represented by green squares $(\square)$ and black plus signs $(+)$, respectively with the appropriate projection of the unit normal of the lobe - plasma sheet boundary for that event
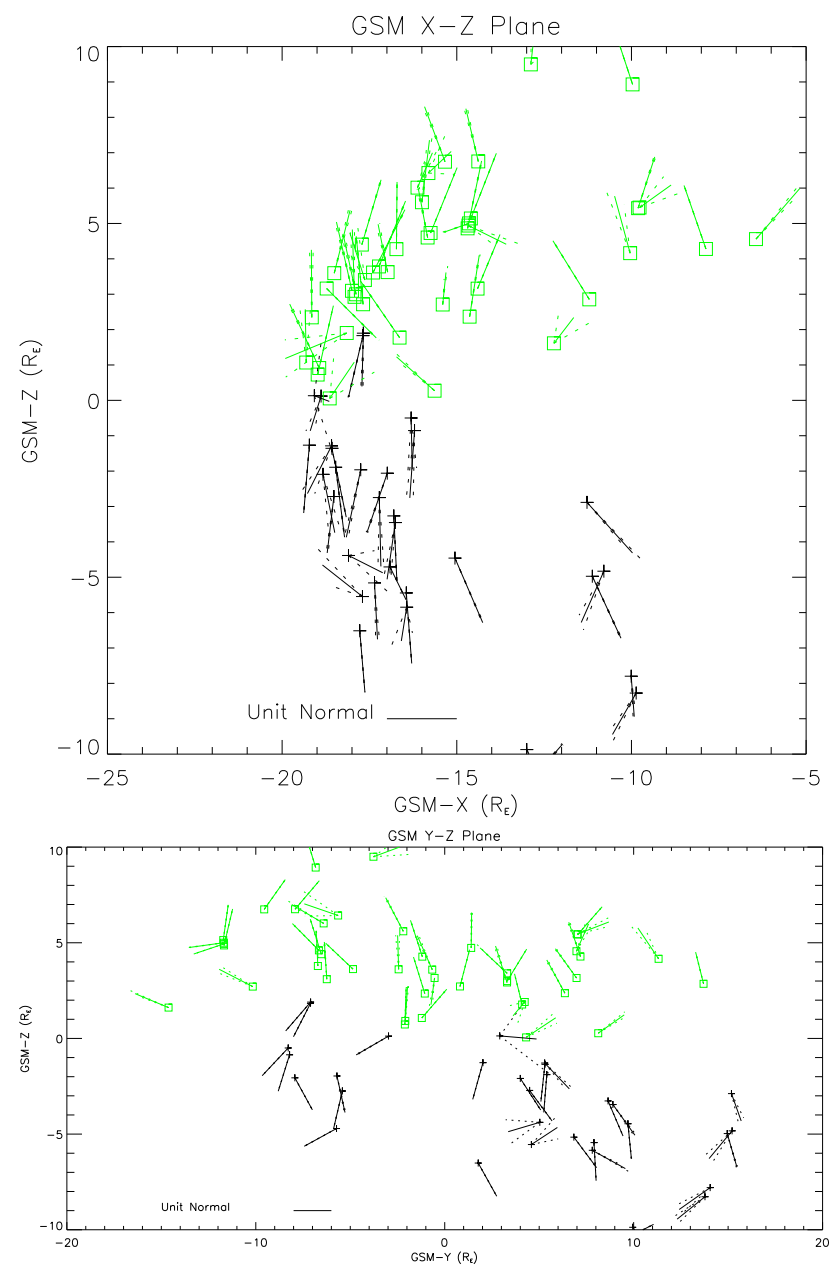

Fig. 12. For each passage of the spacecraft from the lobe into the plasma sheet on the GSM-YZ and GSM-XY planes, the projection of the lobe - plasma sheet boundary normal vector on that plane is shown, together with an estimate of the error associated with the spacecraft spin (dotted line). A unit normal entirely in that plane is shown for reference. In the north lobe the trend is for expansion normals to point away from neutral sheet and have a negative GSM-Y component. In the south lobe there are two trends: in the pre-midnight region the vectors have a positive GSM-Y component and in the post-midnight region they have a negative GSM-Y component.

is shown. In the Northern Hemisphere the normal vectors (green vectors) in the GSM-YZ plane (lower panel) usually point away from the expected location of the current sheet (i.e. have a positive GSM-Z component) and tend to have negative GSM-Y components across the whole width of the magnetotail. There are notable exceptions at high GSM-Z values, where some vectors have a strong positive GSM-Y component. In the Southern Hemisphere (black vectors) there are two differing trends: the vectors have a slight tendency to a negative GSM-Y component in the post-midnight sector and a strong positive GSM-Y component in the premidnight sector. 

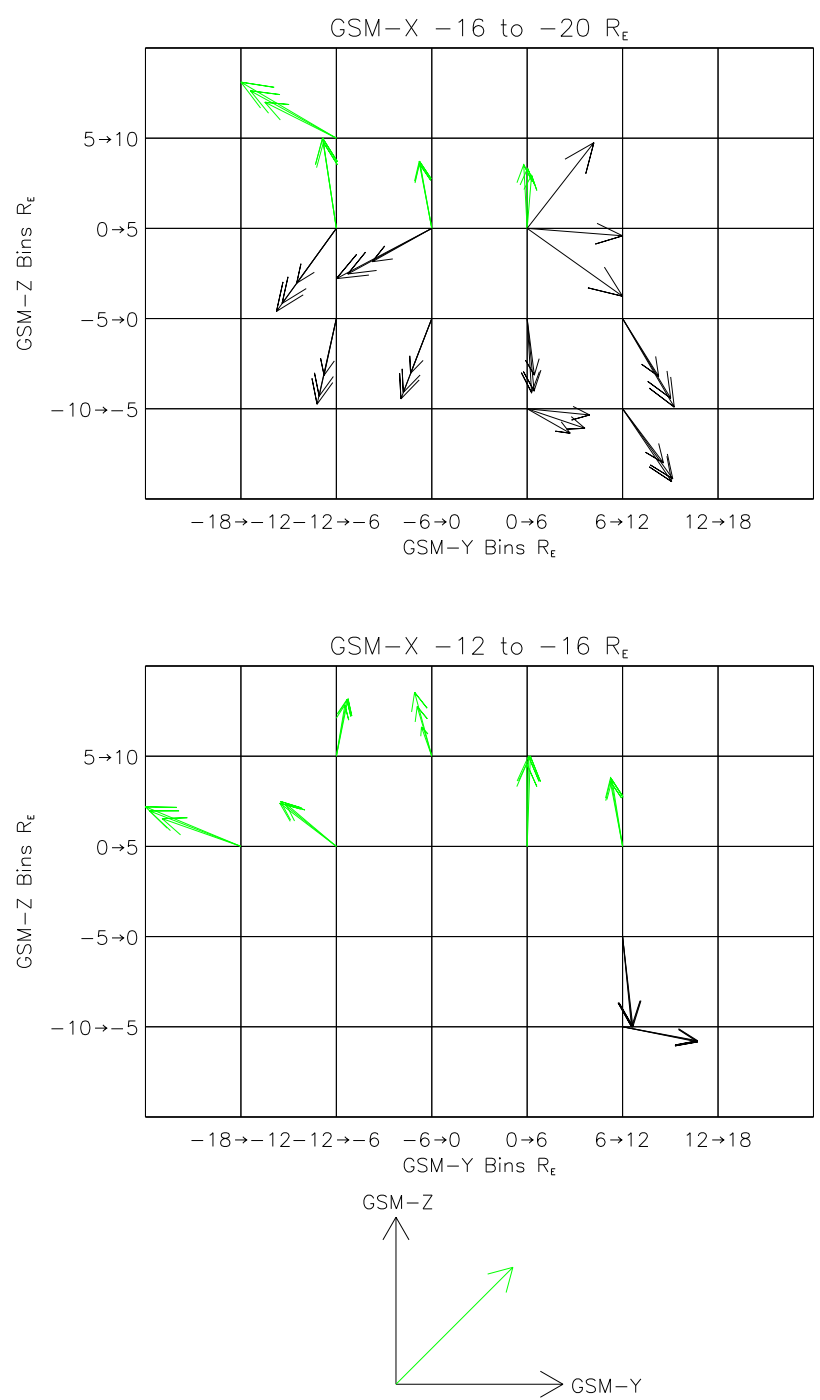

Fig. 13. The projection of the average unit normal of the boundary between the expanding plasma sheet and the lobe in the GSM-ZY direction is shown as a function of position in the GSM-ZY plane for the north lobe (green vectors) and south lobe (black vectors). Error bars, calculated from the extreme errors on the individual vectors, are plotted when more than one normal vector was used for the averaging.

Again, however, clear exceptions exist at all GSM-Z values. The data in the GSM-XZ plane (top panel) shows that there is a strong variation in the GSM-X component of the expansion vectors in both hemispheres. As for the case of the plasma sheet thinnings, the expansion set data will now be binned to provide further clarity.

Figure 13 shows the GSM-ZY projection of the binned normal vector averages as a function of spacecraft position in the GSM-YZ plane for two of the four bins along the GSM$\mathrm{X}$ axis $\left(-20 \leq \mathrm{X}_{\mathrm{GSM}} \leq-16 \mathrm{R}_{E}\right.$ and $\left.-16 \leq \mathrm{X}_{\mathrm{GSM}} \leq-12 \mathrm{R}_{E}\right)$ for those boundaries associated with the spacecraft passing from the lobe into the plasma. The format and binning used is the same as for Fig. 10.
In both GSM-X bins the expansion vectors in the Northern Hemisphere (green vectors) typically have a negative GSM$Y$ component increasing in significance towards the dawn flank, usually with a positive GSM-Z component. For expansions in the south lobe (black vectors) there are two significant trends in the $-20 \leq X_{\mathrm{GSM}} \leq-16 \mathrm{R}_{E}$ bin, where the vectors in the pre-midnight sector have a positive GSM-Y component, whereas in the post-midnight sector they have a negative GSM-Y component. There are only two data bins in the $-16 \leq \mathrm{X}_{\mathrm{GSM}} \leq-12 \mathrm{R}_{E}$ range that contain expansion vectors, and these exhibit the same trend of having a positive GSM-Y component with a negative GSM-Z component in the dusk sector.

Figure 14 shows the GSM-XZ projection of the binned normal vector averages for each lobe as a function of spacecraft position in the GSM-XY plane for those boundaries associated with the spacecraft passing from the lobe into the plasma sheet, using the same format as Fig. 11.

In the Northern Hemisphere, the expansion vectors (green) exhibit a slight to moderate positive GSM-X component in the post-midnight sector compared to a variety of directions and significance in the pre-midnight sector. In the Southern Hemisphere (black vectors) along the $-20 \leq \mathrm{X}_{\mathrm{GSM}} \leq-16$ bin the post-midnight sector shows a slight negative GSM-X component with the pre-midnight sector exhibiting no significant GSM-X component. The vectors in the dusk sector show major variation in the GSM-X component of expansion.

The distribution of normal velocity magnitudes is shown in Fig. 15 for the 66 cases of plasma sheet thinnings. The data was binned into $10 \mathrm{~km} \mathrm{~s}^{-1}$ bins. The absence of a 0 to $10 \mathrm{~km} \mathrm{~s}^{-1}$ bin is due to the event selection criteria eliminating those events subject to large timing errors and for where the assumptions may not be valid. Outliers beyond the $180-190 \mathrm{~km} \mathrm{~s}^{-1}$ bin have also been excluded. The average normal velocity of thinnings is $\sim 44 \pm 32 \mathrm{~km} \mathrm{~s}^{-1}$ (errors from one standard deviation). Similarly, the expansion normal velocities magnitudes are shown in Fig. 16 and are binned in the same way. The average normal velocity along the normal direction of the boundary motion was found to be $\sim 61 \pm 37 \mathrm{~km} \mathrm{~s}^{-1}$ for expansions (error taken as one standard deviation).

An assessment of whether the expansion speed of the plasma sheet over the Cluster quartet scales with the magnitude of the associated substorm auroral electrojet (AE) index was made. The AE index was averaged over a period of $30 \mathrm{~min}$, centred on the time of each boundary crossing, and then rounded to the nearest $50 \mathrm{nT}$, to provide an indicator of the intensity of the associated substorm. At the time of publication only numerical data for the 2001 provisional AE index were available from the World Data Centre (WDC-C2, Kyoto); accordingly, the averages for the 2002 events were assessed by visual inspection of the AE Quick Look data plots available from the World Data Centre.

The average $\mathrm{AE}$ (in $\mathrm{nT}$ ) and the plasma sheet expansion velocity magnitude (in $\mathrm{km} \mathrm{s}^{-1}$ ) along the normal direction of the plasma sheet - lobe boundary are shown in Fig. 17. 
The Pearson's product moment correlation coefficient for the plotted data is -0.06 , indicating little association between the normal velocity component and the AE index. It should be noted that the occurrence of strong substorms (i.e. AE greater than $450 \mathrm{nT}$ ) are relatively uncommon in this data set with an average AE closer to $350 \mathrm{nT}$. Limited observations of high AE substorms may skew the distribution.

To assess the effect of the variation in IMF direction on the data set, the relation between IMF $\mathrm{B}_{Y}$ (in nT) and the GSM-Y component, $\mathrm{n}_{Y}$, of the expansion normal unit vector is shown in Fig. 18. Where possible the IMF data was obtained by averaging the solar wind velocity as measured by the ACE/SWE experiment over the period 50 to $70 \mathrm{~min}$ before the expansion event was observed at Cluster. The average velocity was used to obtain an average lag time with reference to the ACE-Earth distance. The values for the IMF were then found by lagging the magnetic field data from MFI by this averaged time lag. When data gaps were present the data were visually inspected to obtain the appropriate magnetic field components. A positive $\mathrm{n}_{Y}$ indicates expansion in the positive GSM-Y direction, i.e. towards dawn. There are more events with a positive IMF $\mathrm{B}_{Y}$ component, but these occur with a wide spread in the normal direction. Consequently, there is no simple relationship between the IMF $\mathrm{B}_{Y}$ component and the normal GSM-Y component.

An attempt to determine if the plasma sheet - lobe boundary decelerates with distance from the expected neutral sheet location was also made. The average neutral sheet location was taken to be that modelled by Dandouras (1988) and the normal component of the velocity against the distance of the spacecraft quartet from the neutral sheet, $d Z\left(\mathrm{R}_{E}\right)$, is plotted in Fig. 19. A decelerating boundary would result in those observations made further away from the neutral sheet being associated with slower boundaries. However, there appears to be a significant variability of the normal velocity component with distance up to $8 \mathrm{R}_{E}$ away from the neutral sheet, implying that the plasma sheet can expand with large velocities and that these can be maintained over a large distance.

\subsection{Paired and multiple events}

As the magnetospheric substorm goes through its cycle, the plasma sheet is expected to first thin, and then expand over the spacecraft. As a result of this cyclic nature we would expect that in the available data set all thinnings are followed by an expansion. Naturally, due to the variable extents of plasma sheet expansion in the north-south direction and with extra complications due to spacecraft and instrument operations, we do not have a complete data set of paired events. For those events that have both the thinning-expansion components, as in the example shown in Fig. 5, we are able to compute the ratio of the thinning to expansion normal velocities to examine, for example, whether or not a rapid thinning is followed by a rapid expansion.

In total there are 29 paired plasma sheet thinning and expansion events defined as a growth phase type thinning followed by an onset type expansion located within $15 \mathrm{~min}$ of

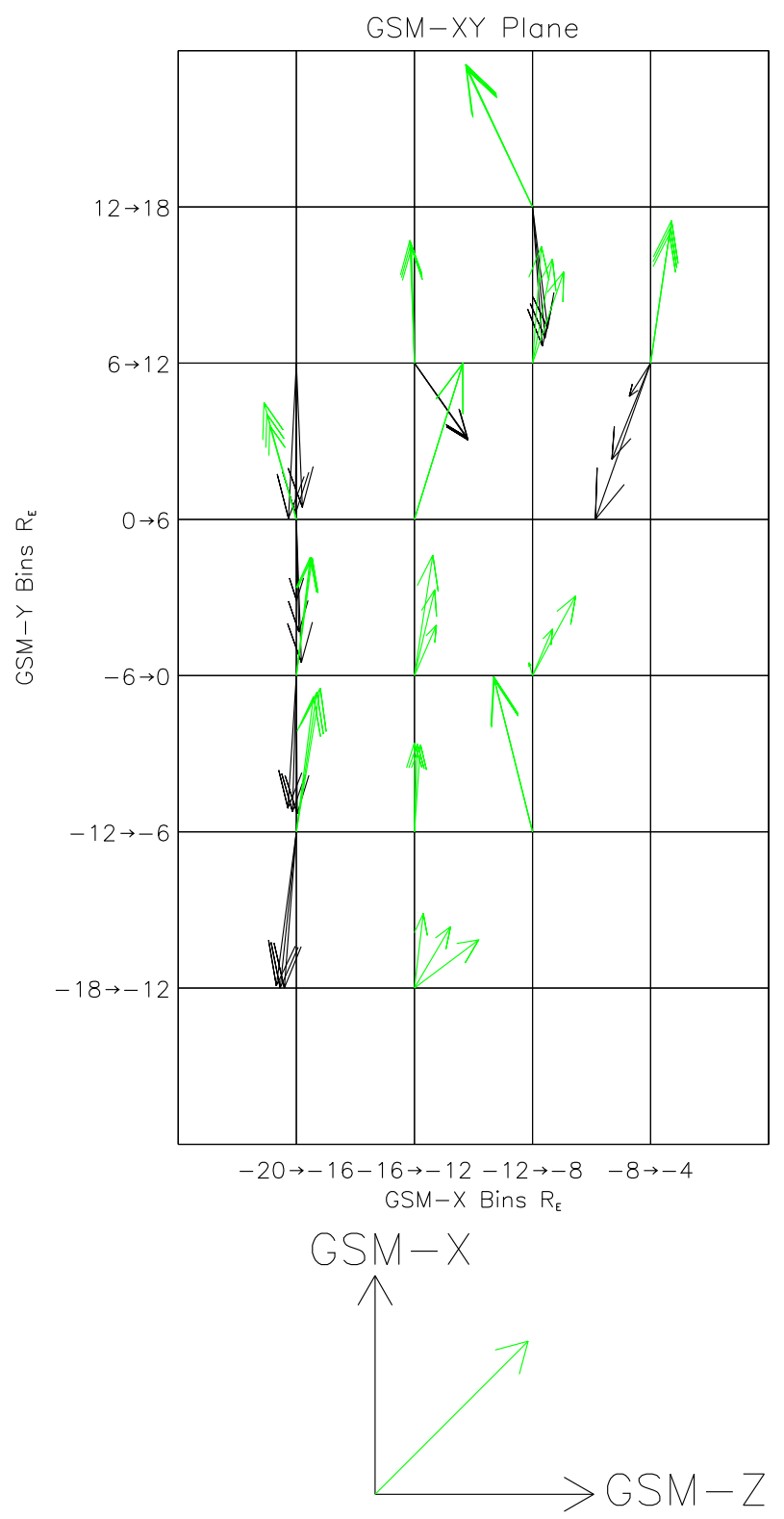

Fig. 14. The projection of the average unit normal of the boundary between the expanding plasma sheet and the lobe in the GSM-ZX direction is shown as a function of position in the GSM-YX plane for two GSM-Z bins. Plasma sheet to lobe crossings are marked in green and black vectors for the north and south lobes, respectively. Error bars, calculated from the extreme errors on the individual vectors, are plotted when more than one normal vector was used for the averaging. In the pre-midnight sector of the Northern Hemisphere the expansions, on average, have a negative GSM-X component that is reversed on the post-midnight sector. Bar a couple of exceptions, the expansion vectors in the south lobe have a GSM-X component ranging from minimal to strongly positive.

an $\mathrm{AE}$ intensification (defined as an increase of at least $50 \mathrm{nT}$ above a background $\mathrm{AE}$ of at least $100 \mathrm{nT}$ ) that are clearly defined in the data set, allowing the timing analysis to be used. Of these there are 16 events were the expansion velocity is greater than the thinning velocity. There are two outlying 


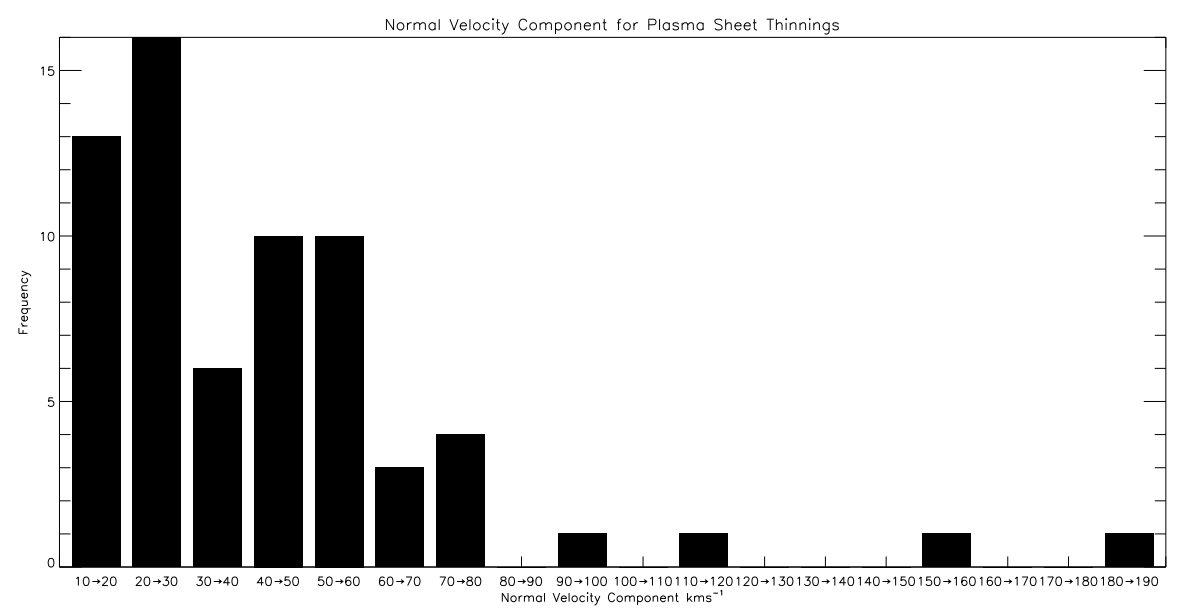

Fig. 15. A bar chart of the normal velocity component of all plasma sheet-lobe boundaries passing over the spacecraft associated with plasma sheet thinning. The speeds are binned into $10 \mathrm{~km} \mathrm{~s}^{-1}$ bins. The average is $48 \pm 38 \mathrm{~km} \mathrm{~s}^{-1}$.

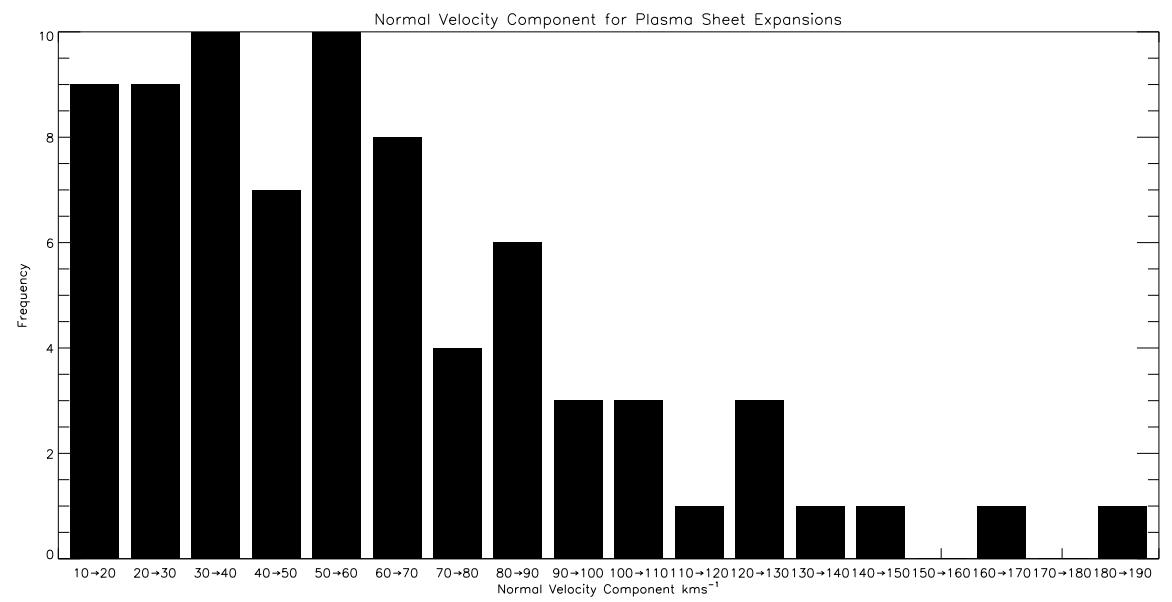

Fig. 16. A bar chart of the normal velocity component of all plasma sheet-lobe boundaries passing over the spacecraft associated with plasma sheet expansion. The speeds are binned into $10 \mathrm{~km} \mathrm{~s}^{-1}$ bins. The average is $60 \pm 37 \mathrm{~km} \mathrm{~s}^{-1}$.

events with a thinning to expansion normal velocity ratio of $\sim 4.8$ and $\sim 16.5$. The average thinning over expansion ratio of the normal velocity components, including the outliers, is 1.58 , implying that on average the expansion velocity is slower than the thinning velocity. Excluding the outliers this ratio drops to 0.91 , suggesting that on average the expansion velocity is similar to the thinning velocity. With reference to the averages presented for the expansion and thinning normal velocity component, we would place this average ratio to be approximately 0.8 .

\section{Summary and discussion}

The observed expansions of the plasma sheet over the Cluster spacecraft for the first time provide information on the direction of the propagation of expansion and thus may indicate the relative location of the onset region. Our expectations were that in the GSM-ZY plane, expansions solely in the Z-direction indicate that the plasma sheet expands uniformly along the whole tail (Fig. 20a). Alternatively, a negative X-component shows that the plasma sheet expands from the dipolar magnetic field region as the excess open flux is removed and the pressure is released (Fig. 20b). Finally, a positive $\mathrm{X}$ component of the expansion normal vector perhaps implies that the onset of expansion is initiated tailward of the spacecraft and, therefore, allows expansion to begin and propagate earthward (Fig. 20c).

In the GSM-ZY plane, as shown in Fig. 13, the projection of the expansion vectors should trace back to the average location of onset if the open flux is evenly distributed throughout the tail lobes and the expansion that occurs at the onset region is allowed to propagate radially away from the onset region in the GSM-YZ plane. The plasma sheet can also be tilted due to interplanetary magnetic field conditions (e.g. Akasofu, 1986; Owen et al., 1995). The complexity of the tail dynamics superposed upon the variable location and ori- 


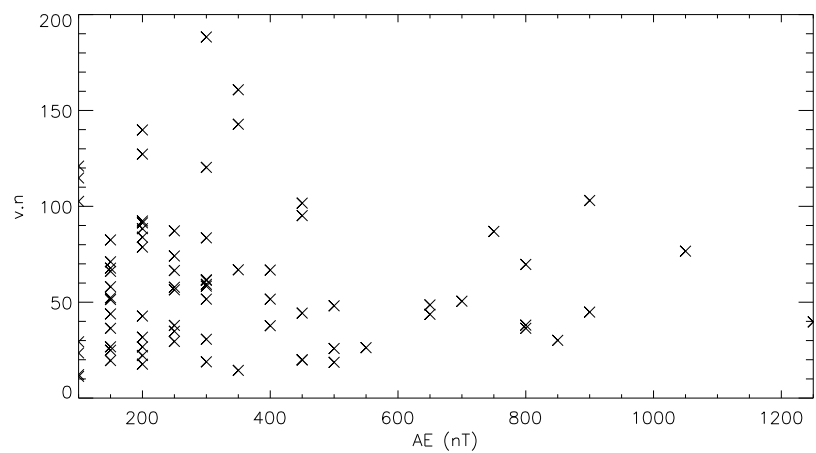

Fig. 17. The normal velocity component of the plasma sheet expansions plotted against average $\mathrm{AE}$ conditions to test for substorm size dependence on the expansion normal velocity. The average substorm condition in this data set has an AE of $\sim 350 \mathrm{nT}$. There exists no correlation with a Pearson's product moment correlation coefficient of -0.06 .

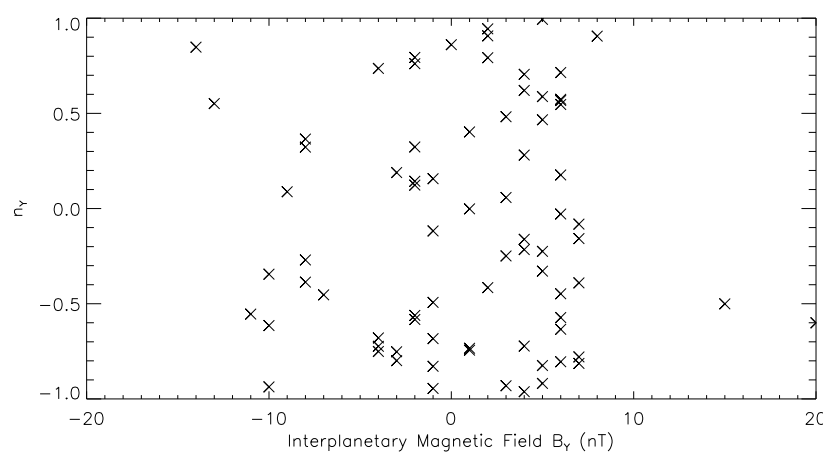

Fig. 18. The GSM-Y component of the normal unit vector is compared to the IMF-Y (GSM) component to test for correlation. Lagged magnetic field data from ACE were used to obtain the IMF-Y input. No simple relationship exists between the IMF-B $Y$ and the normal vector of the expansion.

entation of the plasma sheet adds to the difficulty in drawing definitive conclusions.

On average, the expansion of the plasma sheet occurs in the expected GSM-Z direction: northward in the north lobe and southward in the south lobe. There are notable exceptions that could indicate that the neutral sheet and plasma sheet have more complicated cross tail geometry. Indeed, recent observations by Cluster have shown that the current sheet can carry $1 \mathrm{R}_{E}$ amplitude oscillations with a quasiperiod of $\sim 3 \mathrm{~min}$ in which the vertical speed of the flapping motion is in excess of $100 \mathrm{~km} \mathrm{~s}^{-1}$ (Sergeev et al., 2003). These waves were observed on a tilted current sheet $\sim 45^{\circ}$ in the GSM-YZ plane. The complexity shown in the motion of the current sheet may translate through to the entire plasma sheet. Recent work by Zhang et al. (2002) used Cluster data to demonstrate the variation in the current sheet on short times scales. Both the Zhang et al. (2002) and Sergeev et al. (2003) studies link the complex tilting of the neutral sheet to dawn-to-dusk wave propagation superimposed onto the already tilted neutral sheet.

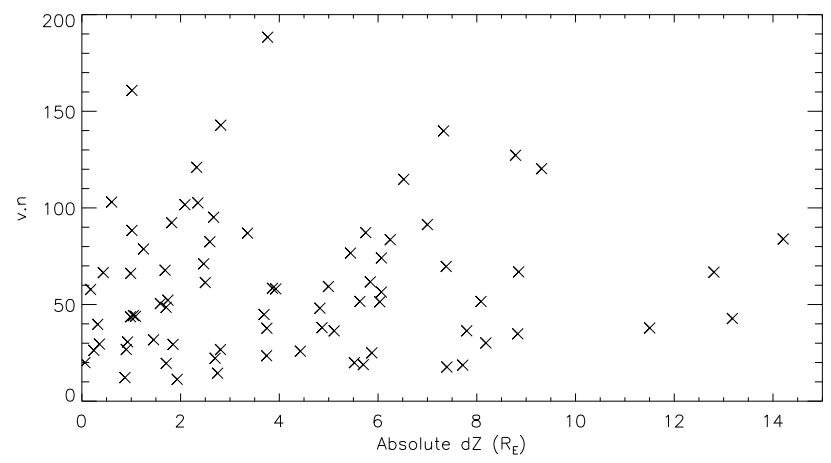

Fig. 19. The normal velocity component plotted against distance from the neutral sheet modelled by Dandouras (1988) for plasma sheet expansions. The variability of the normal velocity component with $d Z$ suggests that the velocity at which the boundary moves is sustained, or is not decelerated, after its initial acceleration.

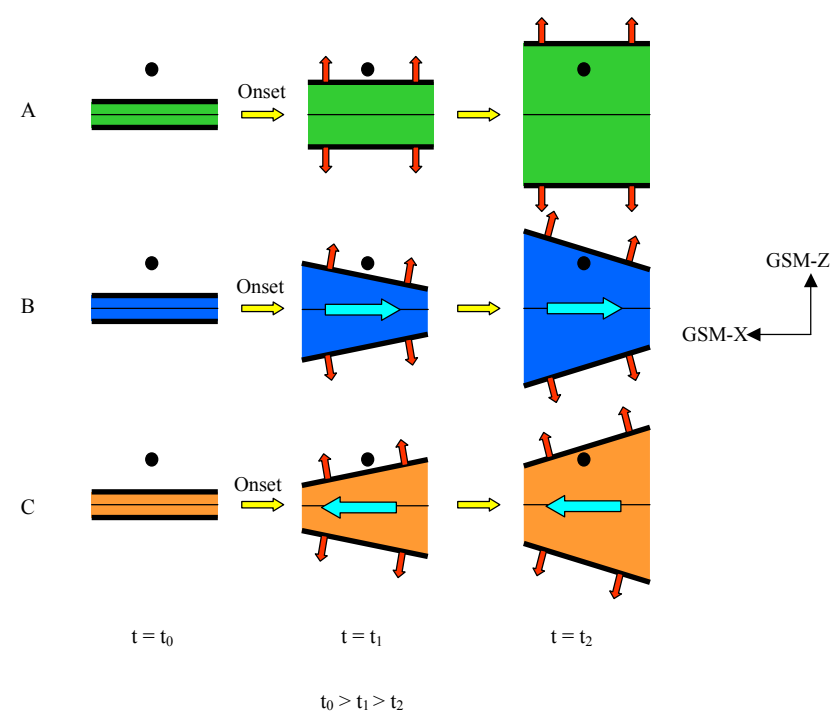

Fig. 20. The normal vectors (red) of the lobe - plasma sheet boundary as the plasma sheet expands: (A) uniformly down the $\mathrm{X}$ axis; (B) tailwards from near the Earth; (C) and, earthwards from tailward of the spacecraft. For each case, the plasma sheet prior to onset and its subsequent expansion is shown. Any propagation direction of the expansion is also shown (cyan). The spacecraft quartet is shown as a black circle.

In the dusk sector of the south lobe there is predominately an expansion in the positive GSM-Y direction and, complementarily, a negative GSM-Y component in the dawn sector. This is consistent with the site of onset occurring midtail, with expansion occurring dawnward and duskward. Figure 21 illustrates this mode of expansion in the GSM-YZ plane as viewed from the Earth looking tailward.

There is a slight negative GSM-Y component in most north lobe to plasma sheet expansions, increasing in significance towards the dawn flank. Towards dawn, this would be consistent with expansion propagating from the central GSM-Y region of the plasma sheet. 

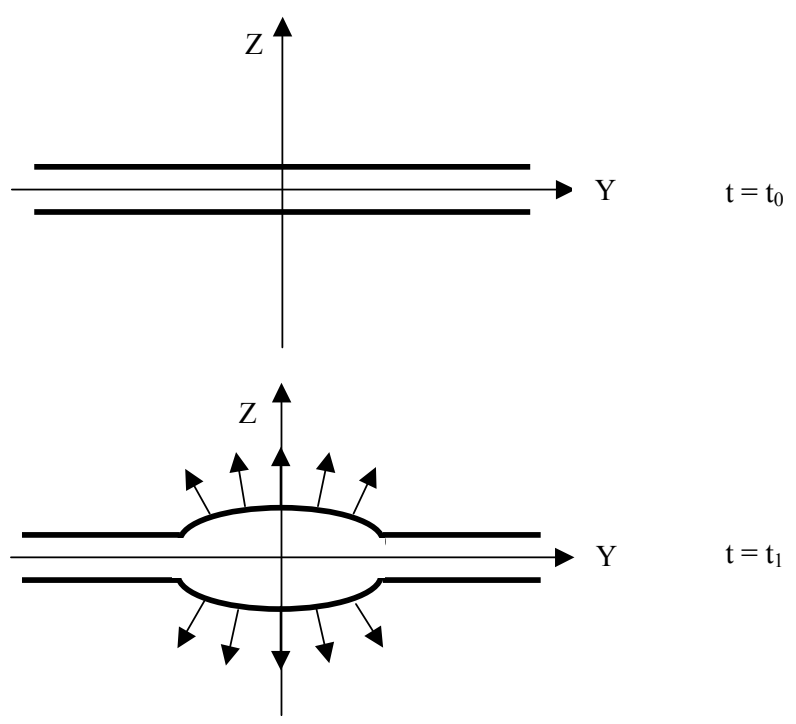

$\mathrm{t}=\mathrm{t}_{1}$

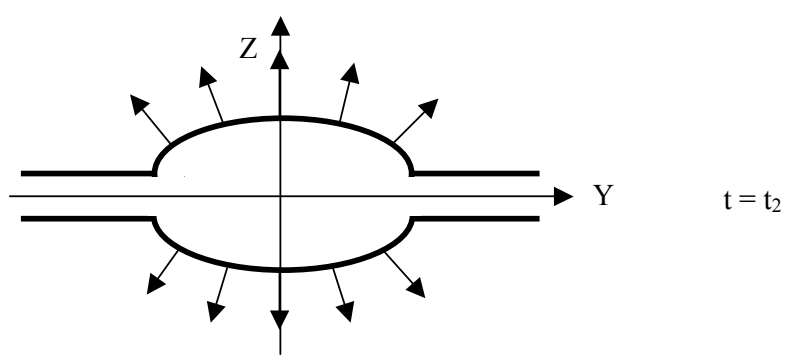

$\mathrm{t}_{0}>\mathrm{t}_{1}>\mathrm{t}_{2}$

Fig. 21. An illustration of a model expansion of the plasma sheet in the GSM-YZ plane as viewed from the Earth looking tailward. The onset region for the expansion is in the central GSM-Y region.

A lack of observations towards dusk prevents comments on the expansion of the plasma sheet in this region. If the expansion occurs principally in the normal of this plane, an extreme tilt of the current and plasma sheet (e.g. Akasofu, 1986) may cause the expansion to have a stronger GSM-Y component which otherwise looks like an anomalous boundary. The events that have a large component along the GSM$\mathrm{Y}$ axis during entry into the plasma sheet are possibly attributed to a dramatic tilting of the neutral sheet and plasma sheet structure (Sergeev et al., 2003).

Periods of repeated boundary crossings with varying normal directions indicate that the plasma sheet dynamics are highly complicated. These variations could be triggered by, for example, changes in the structure of the magnetosphere by variation in the IMF (Akasofu, 1986) or inherent plasma sheet inhomogeneities.

In most of the data bins there is no major GSM-X component of the expansion normal, as expansion in the GSM-YZ plane is dominant. No strong evidence has been obtained to favour either direction sense of the propagation of the plasma sheet expansion in the GSM-X direction; therefore, neither mode of onset (current-disruption or near-Earth reconnection) can be said to impose influence on this study. Simultaneous measurements from close to Cluster apogee and from the near-Earth are probably required to examine the propagation of the expansion. The possibility of X-line observations by Cluster at $\sim-20 \mathrm{R}_{E}$ also complicates the observation of plasma sheet thinning and expansion and requires careful analysis to handle any complex topography near the expected neutral line region.

The explosive nature of the expansion phase is shown with velocities averaging $\sim 61 \pm 37 \mathrm{~km} \mathrm{~s}^{-1}$. This is approximately $20 \mathrm{~km} \mathrm{~s}^{-1}$ faster than that presented by Pytte et al. (1978). Although our estimate is averaged over all distances away from the neutral sheet, the scatter of normal velocity component against distance calculated from the model neutral sheet by Dandouras (1988), shown in Fig. 19, provides no evidence of acceleration or deceleration of the plasma sheet - lobe boundary. Of note is the large standard deviation from the mean velocities: plasma sheet thinnings can be greater than the velocities associated with the subsequent plasma sheet expansion.

The observed thinnings of the plasma sheet show the expected GSM-Z direction, although there is significant variety in the GSM-Y components across the tail. This implies that there is a lot of variation in the tail of the growth phase and the variation in the thinning normal may be because of twisting of the tail or a magnetic flux being added non-uniformly across the tail, for example. The possibility of post-flow associated thinning of the plasma sheet being included in this study exists, but is believed to be minimised by the AE selection criteria.

The difference in the speeds of the thinnings to expansions is understandable in terms of the explosive nature of substorm onset, although the details of the individual pairs of thinning to expansion speeds shows that thinnings speeds can be comparable to expansion speeds.

\section{Conclusions}

In the 2001 and 2002 magnetotail passes, 77 expansions of the plasma sheet over the Cluster quartet were clearly observed. The direction and normal velocity were calculated for these events, and the principle observation was the main direction of the expanding plasma sheet in the GSM-YZ plane. There is insufficient evidence in this data set to determine whether the expansion of the plasma sheet begins at the region associated with current disruption and propagates tailward or in the vicinity of the near-Earth neutral line and then propagates tailward. Higher temporal resolution or multiple observation sites along the axis of the magnetotail are required. The presented data confirms the expected complexity of the plasma sheet and its dynamics, and high degrees of tilting or undulations of the plasma sheet in the GSM-YZ plane may explain the range of directions of the expansion normal. The more variable nature of the thinning normal may be explained by tilting of the magnetotail during the growth phase 
or by the non-uniform addition of magnetic flux along the cross-section of the tail. A more detailed study of the events, where timings of all the other substorm related phenomena can be obtained, may provide further detail to this study.

Acknowledgements. The authors with to thank the World Data Centre for Geomagnetism, Kyoto, for provisional AE numerical data for 2001 and AE index plots for 2002 and the Cluster Science Data System and the FGM team for the provision of FGM Prime Parameter data. Thanks are also given to N. Ness for the provision of ACE MFI data and to D. J. McComas for the provision of ACE SWE data, both attained through the CDAWeb system. The authors thank the SAMNET team for magnetometer data. SAMNET is a PPARC National Facility operated by Lancaster University. SOPA LoE and SOPA LoP browse data was obtained from LANL and the authors wish to thank Reiner Friedel for his assistance in confirming the data. JPD wished to thank M. G. G. T. Taylor for many discussions on the analysis method. Much appreciation is given to M. Kaiser and the Wind/Waves team for the RAD1 data. JPD and CJO wish to thank PPARC for postgraduate and Advance Fellowship funding, respectively.

Topical Editor T. Pulkkinen thanks A. Ieda and another referee for their help in evaluating this paper.

\section{References}

Akasofu, S.-I. and Chapman, S.: The ring current, geomagnetic disturbances and the Van Allen radiation belts, J. Geophys. Res., 66, 1321-1350, 1961.

Akasofu, S.-I: The development of the auroral substorm, Planetary and Space Science, 12, 273-282, 1964.

Akasofu, S.-I.: Polar and Magnetospheric Substorms, D. Reidel, Norwell, Mass., 1968.

Akasofu, S.-I. and Meng, C.-I: Effects of the IMF on the Plasma Sheet, Planet. Space Sci., 34, 683-688, 1986.

Angelopoulos, V., Coroniti, F. V., Kennel, C. F., Kivelson, M. G., Walker, R. J., Russell, C. T., McPherron, R. L., Sanchez, E., Meng, C.-I., Baumjohann, W., Reeves, G. D., Belian, R. D., Sato, N., Friis-Christensen, E., Sutcliffe, P. R., Yumoto, K., and Harris, T.: Multipoint analysis of a bursty bulk flow event on 11 April, 1985, J. Geophys. Res., A101, 4967-4988, 1996.

Aubry, M. P. and McPherron, R. L.: Magnetotail changes in relation to the solar wind magnetic field and magnetospheric substorms, J. Geophys. Res., 76, 4381-4401, 1971.

Axford, W. I., Petschek, H. E., and Siscoe, G. L.: Tail of the magnetosphere, J. Geophys. Res., 70, 1231-1236, 1965.

Baker, D. N., Bame, S. J., Bian, R. D., Fledman, W. C., Gosling, J. T., Higbie, P. R., Hones, E. W. Jr., McComas, D. J., and Zwicl, R. D.: Correlated dynamical changes in the near-Earth and distant magnetotail regions: ISEE 3, J. Geophys. Res., 89, 3855-3864, 1984.

Baker, D. N., Angelopoulos, V., Baumjohann, W., McPherron, R. L., and Pulkkinen, T. I.: The neutral line model of sunstorms: Past results and present view, J. Geophys. Res., 101, 12975$13010,1996$.

Baker, D. N., Peterson, W. K., Eriksson, S., Li, X., Blake, J. B., Burch, J. L., Daly, P. W., Dunlop, M. W., Korth, A., Donovan, E., Friedel, R., Fritz, T. A., Frey, H. U., Mende, S. B., Roeder, J., and Singer, H. J.: Timing of magnetic reconnection initiation during a global magnetospheric substorm, Geophys. Res. Lett., 29, A2190, 2002.
Bame, S. J., Asbridge, J. R., Felthauser, H. E., Hones, E. W. Jr., and Strong, I. B.: Characteristics of the plasma sheet in the Earth's magnetotail, J. Geophys. Res., 72, 113-130, 1967.

Baumjohann, W., Paschmann, G., and Nagai, T.: Thinning and Expansion of the Substorm Plasma Sheet, J. Geophys. Res., 97, 17 173-17 175, 1992.

Bonnevier, B. R. and Rostoker, G.: A three-dimensional model current system for polar magnetic substorms, J. Geophys. Res., 75, 107-122, 1970.

Coroniti, F. V. and Kennel, C. F.: Changes in the magnetospheric configuration during substorm growth phase, J. Geophys. Res., 77, 3361-3370, 1972.

Cummings, W. D., Barfield, J. N., and Colemanm, P. J.: Magnetospheric substorms observed at synchronous orbit, J. Geophys. Res., 73, 6687-6698, 1968.

Dandouras, J., Rème, H., Saint-Marc, A. and Sauvaud, J. A.: A Statistical Study of Plasma Sheet Dynamics Using ISSE 1 and 2 Energetic Particle Flux Data, J. Geophys. Res., 91, 6861-6870, 1986.

Dandouras, J.: On the average shape and position of the geomagnetic neutral sheet and its influence on plasma sheet statistical studies, J. Geophys. Res., 93, 7345-7353, 1988.

Dunlop, M. W. and Woodward, T. I.: Analysis of thick, non-planar boundaries using the discontinuity analyser, Ann. Geophys., 17, 984-995, 1999.

Fairfield, D. H. and Ness, N. F.: Configuration of the geomagnetic tail during substorms, J. Geophys. Res., 75, 7032-7047, 1970.

Fairfield, D. H.: Solar wind control of magnetospheric pressure (CDAW-6), J. Geophys. Res., 90, 1201-1204, 1985.

Hapgood, M. A., Dimbylow, T. G., Sutcliffe, D. C., Chaizy, P. A., Ferron, P. S., Hill, P. M and Tiratay, X.: The Joint Science Operations Centre, Space Sci. Rev., 79, 487-525, 1997.

Haaland, S. E., Sonnerup, B. U. Ö., Dunlop, M. W., Balogh, A., Georgescu, E., Hasegawa, H., Klecker, B., Paschmann, G., PuhlQuinn, P., Rème, H., Vaith, H., and Vaivads, A.: Four-spacecraft determination of magnetopause orientation, motion and thickness: comparison with results from single-spacecraft methods, Ann. Geophys., 22, 1347-1365, 2004.

Harvey, C. C.: Spatial Gradients and the Volumetric Tensor, in Analysis Methods for Multi-Spacecraft Data, edited by G Paschmann and P. Daly, International Space Science Institute and ESA Publications Division, http://www.issi.unibe.ch, 2000.

Hones, E. W. Jr.: Plasma flow in the plasma sheet and its relation to substorms, Radio Sci., 8, 5879-5892, 1973.

Hones, E. W. Jr., Singer, S., Lanzerotti, L. J., Pierson, J. D., and Rosenberg, T. J.: The magnetospheric substorm of 25-26 August 1967, J. Geophys. Res., 76, 2977-3009, 1971a.

Hones, E. W. Jr., Baker, D. N., Bame, S. J., Feldman, W. C., Gosling, J. T., McComas, D. J., Zwickl, R. D., Slavin, J. A., Smith, E. J., and Tsurutani, B. J.: Structures of the magnetotail at $220 \mathrm{R}_{E}$ and its response to geomagnetic activity, Geophys. Res. Lett., 11, 5-7, 1984a.

Hones, E. W. Jr., Pytte, T., and West Jr., H. I.: Associations of Geomagnetic Activity With Plasma Sheet Thinning and Expansion: A Statistical Study, J. Geophys. Res., 89, 5471-5478, 1984b.

Johnstone, A. D., Alsop, C., Burdge, S., Carter, P. J., Coates, A. J., Coker, A. J., Fazakerley, A. N., Grande, M., Gowen, R. A., Gurgiolo, C., Hancock, B. K., Narheim, B., Preece, A., Sheather, P. H., Winningham, J. D., and Woodliffe, R. D.: PEACE: a Plasma Electron and Current Experiment, Space Sci. Rev., 79, 351-398, 1997. 
Kepko, L., Kivelson, M. G., McPherron, R. L., and Spence, H. E.: Relative timing of substorm onset phenomena, J. Geophys. Res., 109, A04203, 2004.

Kokubun, S. and McPherron, R. L.: Substorm signatures at synchronous altitude, J. Geophys. Res., 86, 11 265-11 277, 1981.

Liou, K., Meng, C. I., Lui, T. Y., Newell, P. T., Brittnacher, M., Perks, G., Reeves, G. D., Anderson, R. R., and Yumoto, K.: On relative timing of substorm onset signatures, J. Geophys. Res., 104, 22 807-22 818, 1999.

Lui, A. T. Y.: Current disruption in the Earth's magnetosphere: Observations and models, J. Geophys. Res., A101, 13 067-13 088, 1996.

Maezawa, K.: Magnetotail boundary motion associated with geomagnetic substorms, J. Geophys. Res., 80, 3543-3548, 1975.

McPherron, R. L.: Growth phase of magnetospheric substorms, J. Geophys. Res., 75, 5592-5599, 1970.

McPherron, R. L., Russell, C. T., and Aubry, M. P.: Satellite studies of the magnetospheric substorm on 16 August 1968, 9, Phenomenological model of substorms, J. Geophys. Res., 78, 31313149, 1973.

Ohtani, S, Creutzberg, F., Mukai, T., Singer, H., Lui, A. T. Y., Nakamura, M., Prikryl, P., Yumoto, K., and Rostoker, G.: Substorm onset timing: The 31 December 1995, event, J. Geophys. Res., 104, 22 713-22 728, 1999.

Owen, C. J., Slavin, J. A., Richardson, I. G., Murphy, N., and Hynds, R. J.: Average Motion, Structure and Orientation of the Distant Magnetotail Determined from Remote Sensing the Edge of the Plasma Sheet Boundary Layer with E $>35 \mathrm{keV}$ Ions, J. Geophys. Res., 100, 185-204, 1995.
Riedler, W., Torkar, K., Rüdenauer, F., Fehringer, M., Pedersen, A., Schmidt, R., Grard, R. J. L., Arends, H., Narheim, B. T., Troim, J., Torbert, R., Olsen, R. C., Whipple, E., Goldstein, R., Valavanoglou, N., and Zhao, H.: Active Spacecraft Potential Control, Space Sci. Rev., 79, 271-302, 1997.

Rostoker, G. D.: Current flow in the magnetosphere during magnetospheric substorms, J. Geophys. Res., 79, 1994-1998, 1974.

Rostoker, G., Akasofu, S.-I., Fosters, J., Greenwald, R. A., Kamide, Y., Kawasaki, K., Lui, A. T. Y., PcPherron, R. L., and Russell, C. T.: Magnetospheric substorms-definitions and signatures, J. Geophys. Res., 85, 1663-1668, 1980.

Rostoker, G.: Phenomenology and physics of magnetospheric substorms, J. Geophys. Res., 101, 12 995-12 973, 1996.

Russell, C. T. and McPherron, R. L.: The magnetotail and substorms, Space Sci. Rev., 15, 205-266, 1973.

Pytte, T., McPherron, R. L., Kivelson, M. G., West, H. I. Jr., and Hones, E. W. Jr.: Multiple-satellite studies of magnetospheric substorms: Plasma sheet recovery and the poleward leap of auroral zone activity, J. Geophys. Res., 83, 5256-5268, 1978.

Sergeev, V., Runov, A., Baumjohann, W., Nakamura, R., Zhang, T. L., Volwerk, M., Balogh, A., Rème, H., Sauvaud, J. A., André, M., and Klecker, B.: Current sheet flapping motion and structure observed by Cluster, GRL, 30, 1327, doi:10.1029/2002GL016500, 2003.

Szita, S., Fazakerley, A. N., Carter, P. J., James, A. M., Trávníek, P., Watson, G., André, M., Eriksson, A., and Torkar, K.: Cluster PEACE observations of electrons of spacecraft origin, Ann. Geophys., 19, 1721-1730, 2001.

Zhang, T. L., Baumjohann, W., and Nakamura, R.: A wavy twisted neutral sheet observed by CLUSTER, Geophys. Res. Lett., 29, 1899, doi:10.1029/2002GL015544, 2002. 